\title{
Large-eddy simulation of low-frequency unsteadiness in a turbulent shock-induced separation bubble
}

\author{
Emile Touber \\ Neil D. Sandham \\ This paper is available from Springer in Theor. Comput. Fluid Dyn. (2009) 23:79-107 \\ DOI 10.1007/s00162-009-0103-z \\ http://www. springerlink. com/content/tu262148132n8867/
}

\begin{abstract}
The need for better understanding of the low-frequency unsteadiness observed in shock wave/turbulent boundary layer interactions has been driving research in this area for several decades. We present here a large-eddy simulation investigation of the interaction between an impinging oblique shock and a Mach 2.3 turbulent boundary layer. Contrary to past large-eddy simulation investigations on shock/turbulent boundary layer interactions, we have used an inflow technique which does not introduce any energetically-significant low frequencies into the domain, hence avoiding possible interference with the shock/boundary layer interaction system. The large-eddy simulation has been run for much longer times than previous computational studies making a Fourier analysis of the low frequency possible. The broadband and energetic low-frequency component found in the interaction is in excellent agreement with the experimental findings. Furthermore, a linear stability analysis of the mean flow was performed and a stationary unstable global mode was found. The long-run large-eddy simulation data were analyzed and a phase change in the wall pressure fluctuations was found to coincide with the global-mode structure, leading to a possible driving mechanism for the observed low-frequency motions.
\end{abstract}

Keywords shock boundary layer interaction - global mode $\cdot$ compressible turbulence $\cdot$ LES $\cdot$ low-frequency unsteadiness $\cdot$ separation bubble $\cdot$ digital filter $\cdot$ inflow turbulence

PACS 47.40.Nm $\cdot 47.40 . \mathrm{Ki} \cdot 47.27 . \mathrm{ep}$

\section{Introduction}

Sixty years after the very first observations, shock-wave/boundary-layer interaction (SBLI) research is still an active and challenging field [9]. Such interactions are of practical interest in a number of external and internal flow problems in aerospace such as airframe design and turbomachinery. In some instances, the interaction can significantly alter the heat exchange and produce important unsteady pressure loads which can greatly shorten the structural lifetime [9]. The two-dimensional interactions most commonly studied are the interaction of a normal/oblique shock wave with a laminar/turbulent flat plate boundary layer and the case of a flow over a bump or over a compression corner. A review of the aforementioned interactions can be found in Adamson and Messiter [3]. The case of an incident oblique shock is historically the least well studied, and is the case on which we focus our attention. A sketch of such an interaction is given in figure 1. For a sufficiently large shock strength, the associated adverse pressure gradient induces a separation of the boundary layer. At the leading edge of the separation bubble, compression waves form the reflected shock, which thus originates further upstream than the idealized inviscid theory would predict. The flow deviation along the downside of the bubble produces an expansion fan, quickly followed by reattachment compression waves. Further downstream, the boundary layer recovers to an equilibrium state after a long relaxation process. A snapshot from the present numerical simulation is provided in figure 2 to illustrate the main structures.

Despite the success of the free-interaction theory to describe the initial stage of the laminar interaction [46, 28], theoretical knowledge of the transitional and turbulent cases is extremely limited. In particular, the low-frequency unsteadiness of the reflected shock, although widely acknowledged, is not fully understood [11]. One peculiarity of the observed reflected-shock unsteadiness is its relative low-frequency compared to the characteristic frequency of the incoming turbulent boundary layer [9]. The mechanism causing the low frequency is the focus of much of today's research on SBLI. So far, the published explanations are mainly of a speculative nature. The most common approach is to try to relate upstream events in the incoming turbulent boundary layer with the shock motion. The idea dates back at least to Plotkin [38], who modeled the shock as being randomly perturbed by upstream disturbances but subject to a linear restoring mechanism, forcing the shock to come back to its initial position. Although this approach was successful at predicting some statistical quantities like the wall-pressure root-mean-square, it does not provide a physical explanation of the low-frequency mechanism and is a purely stochastic approach. Furthermore, correctly capturing the flow-variable standard deviation does not imply that the relevant time scales have been properly resolved or modeled. That being said, there is undoubtedly a correlation between the impact of an eddy into the shock and the shock displacement. This led Andreopoulos and Muck [4] to suggest that the frequency of the shock motion scales on the bursting frequency of the incoming boundary layer. Indeed, Erengil and Dolling [16] have shown that the small-scale motions of the shock are caused by its response to the passage of turbulence fluctuations through the interaction. However, such events occur at higher frequencies than the ones we are interested in and cannot be directly related to the large-scale/low-frequency motions of the reflected shock. 


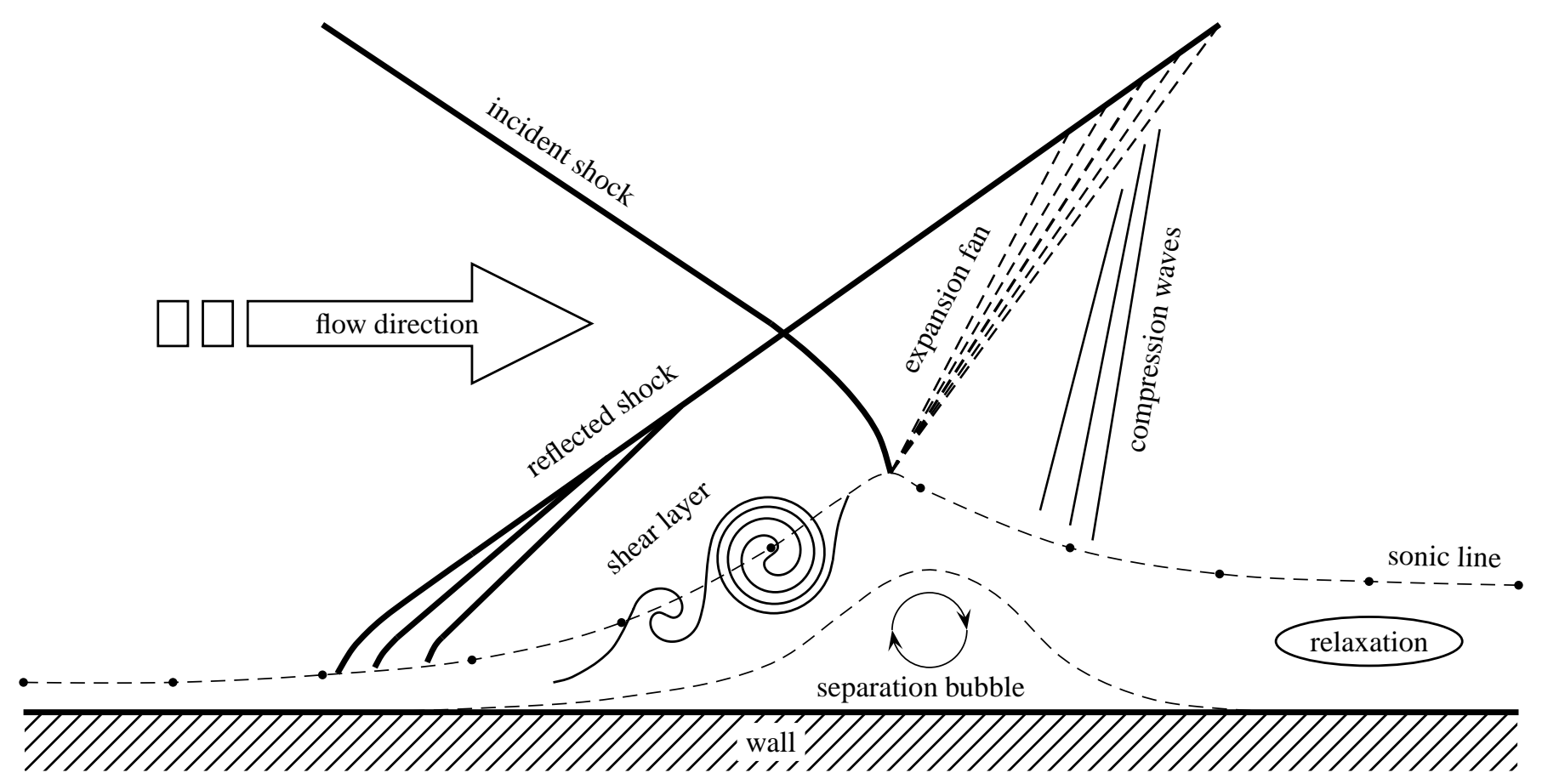

Figure 1: Sketch of the oblique shock / boundary-layer interaction

Ünalmis and Dolling [50] have investigated the correlations in a Mach 5 compression-corner flow between an upstream Pitot pressure and the shock-foot location, and found that an upstream shock position was correlated with higher upstream pressure, and vice versa. It was then argued that the shock position could be driven by a low-frequency thickening and thinning of the upstream boundary layer. Later, Beresh et al. [5] looked at relatively low-frequency correlations in the same compression-corner flow and found significant correlations between upstream velocity fluctuations and the shock motions at 4-10 kHz, one order of magnitude smaller than the characteristic frequency of the large-scale structure of their incoming turbulent boundary layer $\left(U_{\infty} / \delta_{0} \sim 40 \mathrm{kHz}\right.$, where $U_{\infty}$ is the upstream freestream velocity and $\delta_{0}$ the $99 \%$ upstream boundary-layer thickness). Note that in the shock-reflection case we consider in this paper, the upstream boundary-layer characteristic frequency is about $50 \mathrm{kHz}$ while the reported most energetic low-frequency shock motions are at about $0.4 \mathrm{kHz}$ [11].

However, Beresh et al. [5] observe that the "low-frequency thickening/thinning of the upstream boundary layer does not drive the large-scale shock motion". This seems to be in contradiction with the earlier results of Ünalmis and Dolling [50], but later Hou et al. [24] made a similar analysis as Beresh et al. [5] in a Mach 2 compression-corner flow and showed, using conditional averaging, a clear correlation between the shock motion and a thickening/thinning of the upstream boundary layer. It is logical that a change in the upstream mean boundary-layer properties would affect the shock position since a fuller velocity profile would be less prone to separation under the same adverse pressure gradient. This is confirmed, for example, by Beresh et al. [5] who find that their studied correlations improve as they approach the wall.

The aforementioned studies provide clear evidence of a connection between the shock position and the upstream conditionallyaveraged boundary-layer profile. However, the events which are responsible for the substantial differences in the conditionally-averaged profiles had to be clarified, and more importantly, the timescale on which they occur considered with care. Indeed, to be compatible with the shock-motion timescales, those events must be at least an order ten-boundary-layer-thicknesses long. The emergence of timeresolved particle image velocimetry (PIV) approaches made the above considerations possible. For example, Ganapathisubramani et al. [18] have recently reported very long coherent structures of about fifty boundary-thicknesses long (termed "superstructures"), using PIV and Taylor's hypothesis (note that the use of Taylor's hypothesis may be valid as shown by Dennis and Nickels [8]). In their paper, one can find the scaling argument that the low frequency induced by the superstructure scales on $U_{\infty} / 2 \lambda$, where $U_{\infty}$ is the upstream freestream velocity and $\lambda$ the size of the superstructure. In the shock-reflection case we investigate in this paper, we know from Dupont et al. [11] that the energetically significant low-frequency shock oscillations are at about $U_{\infty} / 115 \delta_{0}$, where $\delta_{0}$ is the upstream 99\% boundary-layer thickness. Using the above superstructure-scaling argument, the energetically-significant low frequencies seen in the shock-reflection experiment of Dupont et al. [11] would be associated with structures of size in the order of $50 \delta_{0}$ long, consistent with the value quoted by Ganapathisubramani et al. [18].

Yet, it should be noted that it is uncertain whether such long events are caused by an experimental artifact (such as Görtler-like vortices formed in the expansion section of the wind-tunnel nozzle [5]). Although numerical simulations could, in theory, answer that question, it is not yet possible to perform direct numerical simulations (DNS) which can allow the development of such superstructures and at the same time cover long-enough time series to study the low-frequency shock motions. The reader can refer to the DNS of Ringuette et al. [39] where the authors find long coherent structures up to the maximum domain size tested ( $\left.48 \delta_{0}\right)$. However, it is also unsure whether the recycling/rescaling technique used by the authors could be forcing such structures. In this paper, we will consider the opposite numerical exercise, where we avoid forcing any particular low-frequency/large-wavelength motions and see whether the reflected-shock low-frequency motions can be observed. 
Furthermore, the following two remarks should be considered. First, it must be emphasized that the way the correlation function are built will inevitably govern the level of understanding gained from the resulting correlation values. For example, the correlations mentioned in the above paragraphs are built as follows: the motion of the shock or a predefined separation line is detected and then correlated to an earlier event in the incoming boundary layer, assuming that the upstream event has travelled the separating distance at a constant predefined velocity (usually the local mean velocity). This approach will by construction remove the possibility that the shock motion may be related to a downstream event. Second, such an algorithm always involves in one way or another the choice of arbitrary threshold values, which directly influence the level of correlations seen. For example, Ganapathisubramani et al. [18] define as the separation front the spanwise line from which the velocity is less than $257 \mathrm{~m} \cdot \mathrm{s}^{-1}$ and $187 \mathrm{~m} \cdot \mathrm{s}^{-1}$, due to the difficulty in finding the zero-velocity contour line from the PIV, and the impossibility of using a criterion based on the zero skin-friction contour. With these assumptions, the authors find that the motion of the separation line is correlated to the presence of low- and high- speed regions. The analysis of DNS data allows the study of different possible correlation approaches, which may be difficult or impossible to implement experimentally, and the resulting effect on the interpretation of such correlations. For example, Wu and Martin [55] find that "the streamwise shock motion is not significantly affected by low-momentum structures in the incoming boundary layer". However, using a similar criterion as the one used by Ganapathisubramani et al. [18], the authors found much higher correlation values, similar to the ones found in the experiment. This demonstrates the sensitivity of the correlation techniques in the aforementioned experimental compression-corner investigations. Of course, the ability of numerical simulations to perform time-resolved high-spatial numerical measurements greatly enhances the level of complexity the data analysis can reach. For example, one can look at possible upstream-propagating mechanisms using frequency/wave-number analysis of the wall-pressure distribution (as shown later).

Thus, an alternative (more recent) approach has been to try to relate the low-frequency shock motions to a possible intrinsic mechanism. Recently, Pirozzoli and Grasso [37] performed a DNS corresponding to experiments at Mach 2.3 [11] (mentioned in more detail later in the text), but at a significantly lower Reynolds number than in the experiment. In their paper, the authors argue that the low-frequency originates in an acoustic-feedback-loop mechanism. They suggest that the interaction of the shear-layer coherent structures with the tip of the impinging shock produces acoustic waves which can propagate upstream inside the subsonic region and in turn enhance the shedding of coherent structures at the separation line. This two-dimensional mechanism would then lock itself into a resonant mode and produce the low frequency. However, it is important to note that the integration time obtained by Pirozzoli and Grasso's [37] DNS was much too short to cover any low-frequency oscillation, making the interpretation of their two-point correlation plots subject to caution. The present LES will also address the question of whether or not such upstream propagating acoustic waves can be detected.

The linear stability analysis performed by Robinet [40] shows the existence of a stationary unstable global mode for the laminar interaction case at sufficiently large shock angle but this instability mechanism has not been directly linked to the low-frequency oscillations of turbulent interactions. The present paper will extend the linear stability analysis to the turbulent interaction case and the results will be compared to the LES data, constituting, to our knowledge, the first published attempt of this kind.

Finally, one cannot yet rule out the possibility of experimental and numerical artifacts (like side-wall corner flows in the wind tunnel [13], or the use of recycling/rescaling inflow generators in the simulations [1] ${ }^{1}$ ) which could be at the origin of the low frequencies seen in both numerical simulation and experimental results. In the present LES approach, we try to make sure that this is not the case.

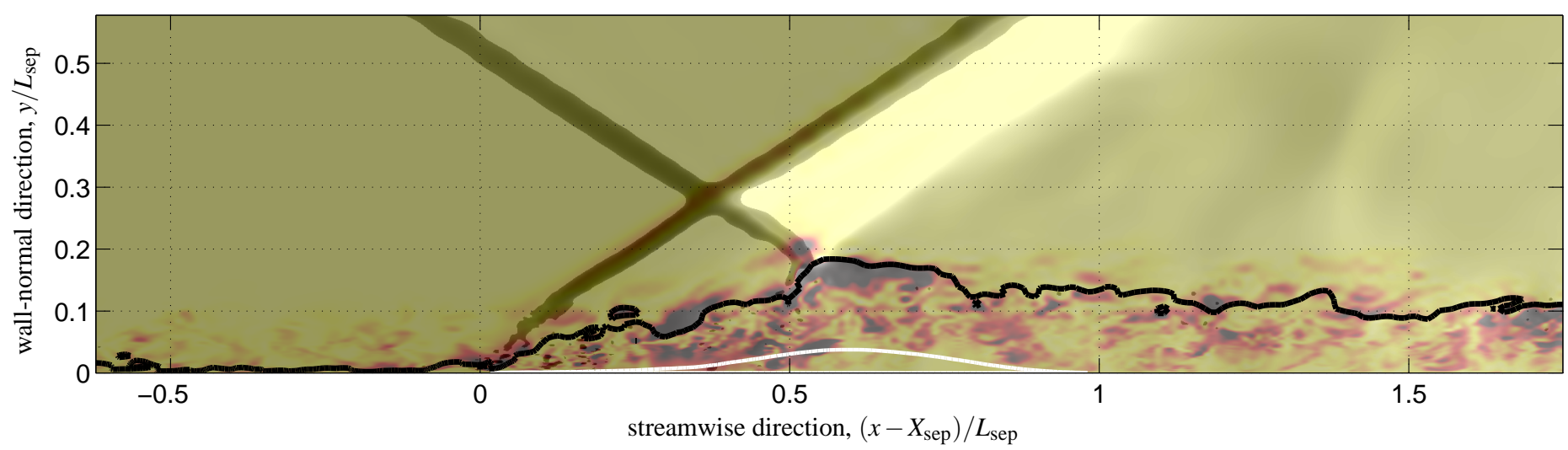

Figure 2: Snapshot from the numerical simulation: shock system (made visible by choosing a velocity-divergence-level range), pressure (black and white) and turbulent kinetic energy (color) fields (both shown on a quadratic scale for highlighting purposes), with sonic line (thick black line) and mean-separation-bubble contour (white line)

The case of a shock-wave generated by a 8 -degree wedge interacting with a turbulent boundary-layer at $\mathrm{Mach}_{2.3}$ and $\mathrm{Re}_{\delta_{1}} \approx 2 \times 10^{4}$ has been experimentally investigated for several years by the IUSTI group in Marseille (France) [13, 11], where $\delta_{1}$ is the boundary-layer displacement thickness (in its compressible-flow formulation) upstream of the interaction. The flow conditions they use are within reach for LES. Indeed, Garnier et al. [19] were the first to perform an LES of that configuration. The authors report relatively good agreement with the mean experimental fields, giving confidence that LES is capable of reproducing the key physics involved in such interactions. However, those authors did not investigate the low-frequency unsteadiness since it would require much longer integration times to cover enough low-frequency oscillations. In fact, the experimental finding is that the period is of the order

\footnotetext{
${ }^{1}$ Private communication with Dr. Eric Garnier, ONERA (2007)
} 
of $115 \delta_{0} / U_{\infty}$ while the LES of Garnier et al. [19] covered only about $88 \delta_{0} / U_{\infty}$. Similarly, the DNS of Pirozzoli and Grasso [37] covered only $25 \delta_{0} / U_{\infty}$, despite the use of a Reynolds number about a third that of the experiment. This is again too short to properly investigate the low frequencies. Although it is in principle possible to perform a DNS of the IUSTI experiment at the true Reynolds number, obtaining a signal at least several low-frequency-period long is still too expensive. The relative success of LES approaches to predict the mean properties of SBLI in past publications [19,32,47] makes it the preferred candidate in order to achieve long time series.

In this paper, we present an LES approach to cover about $10^{4} \delta_{0} / U_{\infty}$ after the initial transient (corresponding to about 90 low-frequency cycles). First, we introduce our LES approach with a particular focus on the inflow generation method, based on the digital filter technique of Klein et al. [30]. Indeed, as mentioned above, the rescaling/recycling technique is believed to potentially affect the SBLI dynamics by introducing a relatively low-frequency tone into the computational box (directly related to the length of the recycling box) which could interfere with the SBLI dynamics. Then, we compare the obtained LES flow statistics against the available PIV data [12]. This is followed by a sensitivity study of the results to the grid resolution, computational domain width and choice of subgrid-scale model. We then present our linear stability analysis approach, and finally analyze the long-run LES data, where we compare our results with the unsteady experimental wall-pressure measurements.

\section{Numerical approach}

\subsection{Governing equations}

After some algebraic manipulation, the approximated form of the filtered dimensionless compressible Navier-Stokes equations (expressed in conservative form) is composed of one continuity equation, three momentum equations and the energy equation:

$$
\begin{gathered}
\frac{\partial \bar{\rho}}{\partial t}+\frac{\partial \bar{\rho} \widetilde{u}_{i}}{\partial x_{i}}=0, \\
\frac{\partial \bar{\rho} \widetilde{u}_{i}}{\partial t}+\frac{\partial \bar{\rho} \widetilde{u}_{i} \widetilde{u}_{j}}{\partial x_{j}}+\frac{\partial \bar{p}}{\partial x_{i}}-\frac{1}{\operatorname{Re}} \frac{\partial \breve{\tau}_{i j}}{\partial x_{j}} \approx-\frac{\partial \sigma_{i j}}{\partial x_{j}}, \\
\frac{\partial \breve{E}_{t}}{\partial t}+\frac{\partial\left(\breve{E}_{t}+\bar{p}\right) \widetilde{u}_{j}}{\partial x_{j}}-\frac{1}{\operatorname{Re}} \frac{\partial \breve{t}_{i j} \widetilde{u}_{i}}{\partial x_{j}}+\frac{1}{(\gamma-1) \operatorname{Re} \operatorname{Pr} M^{2}} \frac{\partial}{\partial x_{j}}\left[\widetilde{\mu} \frac{\partial \widetilde{T}}{\partial x_{j}}\right] \approx-\widetilde{u}_{i} \frac{\partial \sigma_{i j}}{\partial x_{j}}-\frac{1}{(\gamma-1) M^{2}} \frac{\partial}{\partial x_{j}}\left[\bar{\rho} \Theta_{j}\right],
\end{gathered}
$$

where $\rho$ is the fluid density, $u_{i}$ the instantaneous velocity vector, $p$ the pressure, $T$ the temperature and $t$ the time. The streamwise, wallnormal and spanwise directions are denoted by $x, y$ and $z$ respectively. The resolved equation of state, the resolved total energy/pressure relation and the resolved viscous shear-stress relations are:

$$
\begin{gathered}
\bar{p}=\frac{1}{\gamma M^{2}} \bar{\rho} \widetilde{T} \\
\breve{E}_{t}=\frac{\bar{p}}{\gamma-1}+\frac{1}{2} \bar{\rho} \widetilde{u}_{i} \widetilde{u}_{i}, \\
\breve{\tau}_{i j}=\widetilde{\mu}\left(\frac{\partial \widetilde{u_{j}}}{\partial x_{i}}+\frac{\partial \widetilde{u}_{i}}{\partial x_{j}}-\frac{2}{3} \delta_{i j} \frac{\partial \widetilde{u}_{k}}{\partial x_{k}}\right) .
\end{gathered}
$$

The resolved dynamic viscosity $\widetilde{\mu}$ is assumed to follow a power-law dependence with the temperature:

$$
\widetilde{\mu}=[\widetilde{T}]^{\Omega} \quad(\Omega=0.67) .
$$

The overbar and tilde notations were used to denote the spatial-filter and Favre-filter operators, respectively:

$$
\begin{gathered}
\overline{a_{i}}(\mathbf{x})=\int_{\mathscr{D}} G(\mathbf{x}-\mathbf{z} ; \bar{\Delta}) a_{i}(\mathbf{z}) \mathrm{d}^{3} \mathbf{z}, \quad \text { with: } \quad \int_{\mathscr{D}} G(\mathbf{x}-\mathbf{z} ; \bar{\Delta}) \mathrm{d}^{3} \mathbf{z}=1 ; \\
\widetilde{a_{i}}=\frac{\overline{\rho a_{i}}}{\bar{\rho}} .
\end{gathered}
$$

The function $G(\mathbf{x}-\mathbf{z} ; \bar{\Delta})$ is the filter function, with characteristic length scale $\bar{\Delta}$. The integration is performed on a compact subset of $\mathbb{R}^{3}$, denoted $\mathscr{D}$. The usual indicial notation was used, and $\delta_{i j}$ denotes the Kronecker- $\delta$ function, Re the Reynolds number, Pr the Prandtl number (taken to be 0.72), $M$ the Mach number and $\gamma$ the specific heat ratio (taken to be 1.4). The reference values to normalize the flow variables are taken in the potential flow, upstream of the interaction. The reference length scale will vary during the text and will be explicitly defined where it is used. The subgrid-scale (SGS) stress tensor on the right-hand side of equations (2) and (3) is:

$$
\sigma_{i j}=\bar{\rho}\left(\widetilde{u_{i} u_{j}}-\widetilde{u_{i}} \widetilde{u_{j}}\right) \text {, }
$$

and the subgrid-scale Reynolds heat flux on the right-hand side of equation (3) is:

$$
\Theta_{j}=\widetilde{T u_{j}}-\widetilde{T} \widetilde{u_{j}}
$$


Note that the right-hand sides of equations (2) and (3) are incomplete. The list of the neglected SGS terms can be found in Touber and Sandham [49] together with the motivations which led to the above approximate form of the filtered equations. The SGS stress tensor is modeled via the classical eddy-viscosity approach:

$$
\sigma_{i j}-\frac{1}{3} \delta_{i j} \sigma_{k k}=-2 \bar{\rho} v_{t} \widetilde{S_{i j}^{\star}}
$$

where $v_{t}$ is the eddy viscosity and $\widetilde{S_{i j}^{\star}}$ the deviatoric part of the strain-rate tensor computed from the filtered velocity field:

$$
\widetilde{S_{i j}}=\frac{1}{2}\left(\frac{\partial \widetilde{u_{i}}}{\partial x_{j}}+\frac{\partial \widetilde{u_{j}}}{\partial x_{i}}\right), \quad \text { and: } \quad \widetilde{S_{i j}^{\star}}=\widetilde{S_{i j}}-\frac{1}{3} \delta_{i j} \widetilde{S_{k k}} \text {. }
$$

The eddy viscosity is then obtained from the Mixed-Time-Scale (MTS) model by Inagaki et al. [27] which is essentially based on a dimensionally-consistent physical argument relating to the asymptotic behavior of the eddy viscosity as one approaches the wall and the potential flow:

$$
\begin{gathered}
v_{t}=C_{M T S} k_{e s} T_{S}, \\
k_{e s}=\left[\widetilde{u_{i}}-\widetilde{\widetilde{u}_{i}}\right]\left[\widetilde{u_{i}}-\widetilde{\widetilde{u}_{i}}\right], \quad T_{S}^{-1}=\left(\frac{\bar{\Delta}}{\sqrt{k_{e s}}}\right)^{-1}+\left(\frac{C_{T}}{\left|\widetilde{S^{\star}}\right|}\right)^{-1}, \quad \widetilde{\widetilde{u_{i}}}=\frac{\overline{\bar{\rho} \widetilde{u}_{i}}}{\overline{\bar{\rho}}},
\end{gathered}
$$

where the constants $C_{M T S}$ and $C_{T}$ were originally set to 0.05 and 10 by Inagaki et al. [27], based on a priori tests in channel and backward-facing step flow data. In the current implementation of the model, we used:

$$
C_{M T S}=0.03, \quad C_{T}=10,
$$

based on application of the SBLI code to compressible turbulent channel flow. In addition, the dynamic Smagorinsky model [21, 22, 36, 53] was coded based on Vreman's [53] implementation in order to quantify the model effects on the results.

The filter used in the code is a simple top-hat filter with characteristic width equal to the grid spacing. The flow is filtered only in the streamwise and spanwise directions, avoiding issues related to filtering in the stretched-grid direction. When the dynamic Smagorinsky model is used, the test filter is also a top-hat filter with characteristic width two times the grid spacing. Finally, the filter size was defined as:

$$
\bar{\Delta}^{2}=\Delta x \cdot \Delta z
$$

Once the eddy viscosity is obtained (from either one of the above SGS stress tensor models), the SGS heat flux is modeled as:

$$
\Theta_{i}=-\frac{v_{t}}{\operatorname{Pr}_{t}} \frac{\partial \widetilde{T}}{\partial x_{i}}
$$

where $v_{t}$ is taken from the SGS stress tensor model. The SGS turbulent Prandtl number $\operatorname{Pr}_{t}$ should, in theory, be computed dynamically as in Moin et al. [36]. However, we consider it to be constant here (as in Garnier et al. [19]), with $\operatorname{Pr}_{t}=1.0$.

The aforementioned governing equations are solved using a $4^{\text {th }}$-order central spatial differencing scheme for the spatial derivatives and the $3^{\text {rd }}$-order explicit Runge-Kutta scheme to integrate in time. The boundary treatment is also of $4^{\text {th }}$ order [6]. The code makes use of the entropy splitting of the Euler terms and the laplacian formulation of the viscous terms to enhance the stability of the nondissipative central scheme (see Sandham et al. [42]). In addition, a variant of the standard total variation diminishing scheme is used for shock capturing [57], coupled with the Ducros sensor [10]. Periodic boundary conditions are used in the spanwise direction, while the no-slip condition is enforced at the wall, which is set to be isothermal. The top (freestream) and outflow boundaries make use of an integrated characteristic scheme $[48,44]$ in order to minimize unwanted reflections from the computational-box boundaries. The oblique shock is introduced at the top boundary using the Rankine-Hugoniot relationships. The inflow condition is the subject of the following section. Finally, the code was made parallel in all three directions using MPI libraries.

\subsection{Inflow boundary conditions}

DNS and LES of turbulent boundary-layer flows suffer from the need to prescribe accurate three-dimensional and time-dependent inflow-boundary conditions. This is a rather important issue due to the sensitivity of the governing equations to the choice of boundary conditions. Perhaps the most common approach is the rescaling/recycling technique proposed by Lund et al. [33]. This is one of the most accurate approaches since it only requires one empirical relation, introducing almost no inflow transient. However, we argue that this technique suffers from two important drawbacks for the present SBLI study. First, the extension of the method (originally designed for incompressible flows) to compressible flows raises the issue of the rescaling of the thermodynamic variables and the so-called pressure drift (see Sagaut et al. [41] and references therein). Secondly, the recycling nature of the method will, by construction, introduce a distinct low-frequency tone that can interfere with the study of the low-frequency content in SBLI [1] $]^{2}$. All the LES [19, 32, 47] and most of the DNS [54, 1] results available so far on SBLI have used the recycling technique. However, for their DNS, Pirozzoli and Grasso [37] have chosen to use a long domain to simulate the transition to turbulence (note that to achieve this, they forced the flow at the wall over a short streamwise distance). Similarly, for his compression-ramp DNS, Adams [2] used a precursor flat plate DNS where a bypasstransition technique was used. Although very appealing, the overall computational cost of those approaches is prohibitive if we want to

\footnotetext{
${ }^{2}$ Private communication with Dr. Eric Garnier, ONERA (2007)
} 
cover several low-frequency cycles. Alternative techniques are often referred to synthetic turbulence approaches. One of them was developed by Sandham et al. [43] and extended to higher Mach numbers by Li [31]. In this approach, the inflow conditions are prescribed analytically via the introduction of several modes aimed at mimicking the main features of the turbulent flows such as the inner-layer near-wall streaks and their lift up. As with any synthetic turbulence approach, it suffers from the level of approximation introduced at the inflow by producing a long transient during which the flow slowly recovers the modeling errors. Despite that major drawback, synthetic approaches are increasingly becoming popular. Whereas Sandham et al.'s [43] method does not attempt to match the proper statistical moments, other synthetic approaches like the digital-filter (DF) approach [30] are designed to match ad-hoc first and second order statistical moments and spectra. In that case, the lack of proper phase information is responsible for the observed inflow transient.

Table 1: Digital Filter coefficients

\begin{tabular}{lcccccc}
\hline \hline velocity component & \multicolumn{3}{c}{$u$} & \multicolumn{3}{c}{$w$} \\
\hline$I_{x}$ in $\delta_{1}^{\text {vd }}$ units & TBL & SBLI & TBL & SBLI & TBL & SBLI \\
$N_{F_{y}}=2 I_{y} / \Delta y$ (grid points) & $35^{\mathrm{a}}-65^{\mathrm{b}}$ & $20^{\mathrm{a}}-35^{\mathrm{b}}$ & $45^{\mathrm{a}}-85^{\mathrm{b}}$ & $25^{\mathrm{a}}-45^{\mathrm{b}}$ & $30^{\mathrm{a}}-40^{\mathrm{b}}$ & $15^{\mathrm{a}}-20^{\mathrm{b}}$ \\
$N_{F_{z}}=2 I_{z} / \Delta z$ (grid points) & 15 & 20 & 15 & 20 & 30 & 30 \\
\hline \hline a if $y \leq y_{\text {lim }}$, where $y_{\lim }=1 \delta_{1}^{\text {vd }}$ & & & & & \\
b if $y>y_{\text {lim }}$, where $y_{\text {lim }}=1$ & & & & &
\end{tabular}

Before running the SBLI LES, we performed two LES of a flat-plate turbulent boundary layer in order to compare Li's [31] extension of the synthetic turbulence approach of Sandham et al. [43] with a new modified version of Klein et al.'s [30] digital filter approach. The modifications introduced are originally due to Xie and Castro [56] and greatly speed up the original version of Klein et al. [30] by applying the filtering operation only in 2D (rather than in 3D) and correlating the new 2D field with the previous one. In addition, we propose here some modifications to Xie and Castro's [56] work in order to further speed up the technique and extend it to compressible flows. The details of the new digital filter technique are given in appendix A.

The digital-filter coefficients used in both the turbulent-boundary layer test simulation (denoted TBL) and the actual shock/boundary layer interaction simulations (denoted SBLI) are given in table 1. Interestingly, the digital-filter technique was found to be relatively robust to the choice of filter coefficients, which is a desirable feature. However, this is only true as long as the prescribed length scales are at least as large as the integral length scales of the real flow. Failing to meet that requirement can lead to laminarization issues [29, 52] just as when only white noise is added to the flow. The prescribed mean-velocity profile was obtained from the semi-analytical method described in $\mathrm{Li} \mathrm{[31]} \mathrm{and} \mathrm{the} \mathrm{prescribed} \mathrm{Reynolds} \mathrm{stresses} \mathrm{were} \mathrm{obtained} \mathrm{from} \mathrm{an} \mathrm{earlier} \mathrm{simulation} \mathrm{under} \mathrm{similar} \mathrm{flow} \mathrm{conditions} \mathrm{as} \mathrm{the}$ one considered here.

Table 2: Numerical details for the turbulent-boundary-layer simulations

\begin{tabular}{lcccc}
\hline \hline & Streamwise dir. $x$ & Wall-normal dir. $y$ & Spanwise dir. $z$ \\
\hline Domain size & $L_{x}, L_{y}, L_{z}$ in $\delta_{1}^{\text {vd }}$ & 400 & 50 & 20 \\
& $L_{x}, L_{y}, L_{z}$ in $\delta_{0}$ & 28 & 3.5 & 1.7 \\
\hline Number of points $\left(N_{x}, N_{y}, N_{z}\right)$ & 401 & 151 & 81 \\
\hline Grid resolution (wall units) & $\Delta x^{+} \approx 33$ & $\Delta y_{\min }^{+} \approx 1.6$ & $\Delta z^{+} \approx 10$ \\
\hline \hline
\end{tabular}

Both the digital-filter (DF) technique (described in appendix A) and the synthetic turbulence (ST) technique of Sandham et al. $[43,31]$ were compared on a Mach 2 and $\operatorname{Re}_{\delta_{1}^{\text {vd }}} \approx 2500$ turbulent flat-plate boundary-layer flow $\left(\delta_{1}^{\text {vd }}\right.$ is the displacement thickness at the inlet computed from the van-Driest-transformed velocity profile using the incompressible-flow definition of the displacement thickness). The numerical details are given in table 2. Figure 3(a) compares the skin-friction evolution obtained for both the digital filter and the synthetic inlet conditions. The two techniques produce a transient of about 20 boundary-layer thicknesses, as expected for such synthetic approaches [29]. Despite the fundamental differences in the formulation of each technique, the skin friction appears to converge to the expected levels at a similar streamwise location. This indicates that the near-wall region is not sensitive to the prescribed inlet method. This is probably because the near-wall turbulence structures recover the modeling errors fairly quickly and neither of the two approaches perform better there. However, figure 3(b) compares the van Driest velocity profiles (expressed in the classical inner-layer scaling, denoted by the exponent “+”) obtained at $x / \delta_{0} \sim 15$ where some differences are found in the outer-region. Although both approaches produce the expected near-wall asymptotic behavior of a turbulent flow, the wake-region does not appear to be realistic in the case of the synthetic turbulence. This is further seen in the turbulence statistics at the same streamwise station, as shown in figure 4. Figure 4(a) is shown in inner-layer scaling where the compressibility correction of Huang et al. [25] is used for comparison with the DNS data of an incompressible turbulent boundary layer [45]. Figure 4(b) is plotted using the displacement thickness. Both figures exhibit the presence of a second spurious peak in the root-mean-square (RMS) profiles for the synthetic turbulence method. This unexpected distribution of the turbulence energy is due to the presence of a low-frequency, large-wavelength mode, which was introduced at the inlet, and is found to survive for long streamwise distances, even up to the outflow boundary, but eventually converging to the profile obtained with the digital filter. The ability of the last outer mode to survive for such distances was not observed in Sandham et al. [43]. We speculate that this is due to the higher Mach and Reynolds numbers used here, potentially stabilizing this outer mode, whereas the method was found to be successful in a subsonic boundary layer. 
With respect to the SBLI simulations, we clearly do not wish to force a particular low-frequency/long-wavelength mode as this could directly impair the low-frequency study in the interaction. This is the main reason for choosing the digital filter, since it is able to produce realistic inflow conditions with the guarantee to avoid any cyclic pattern. In fact, the digital-filter formulation is convenient since it provides a direct control on the size of the coherent structures introduced at the inlet. In the present SBLI study, the integral lengthscale used in the exponential correlation function (see equation (28)) is set to be less than $0.6 \delta_{0}$. Of course, larger structures can develop in the domain by the time the flow reaches the interaction. However, since the available domain before the interaction is an order $10 \delta_{0}$ long, no structure longer that about ten boundary-layer-thicknesses long can form upstream of the interaction. Evidences on the absence of any upstream low-frequency forcing are provided later in the paper.

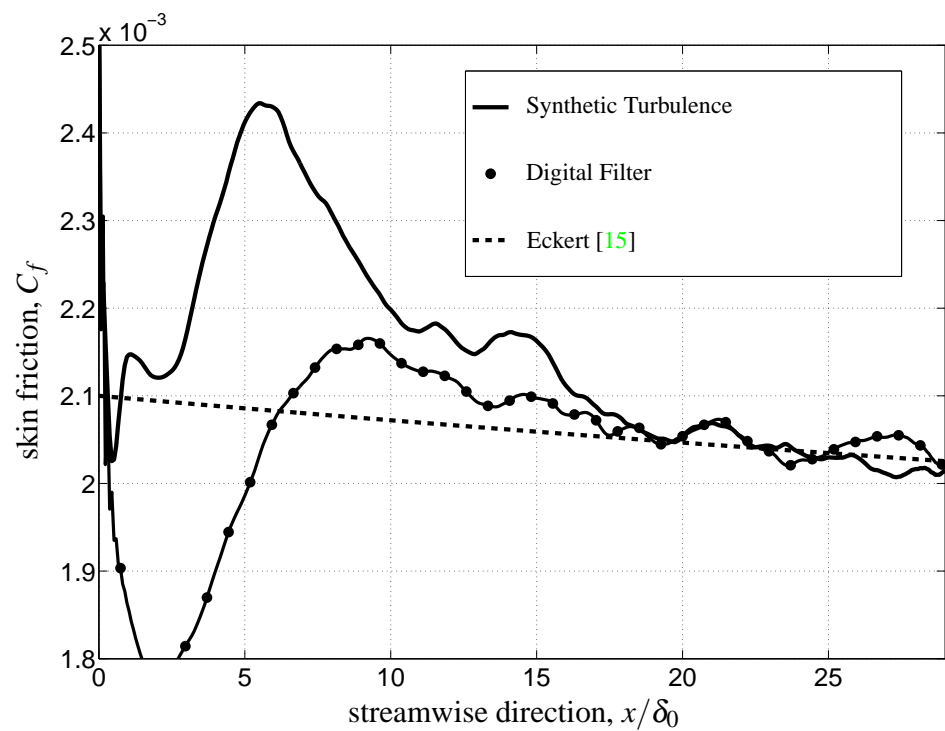

(a) skin friction

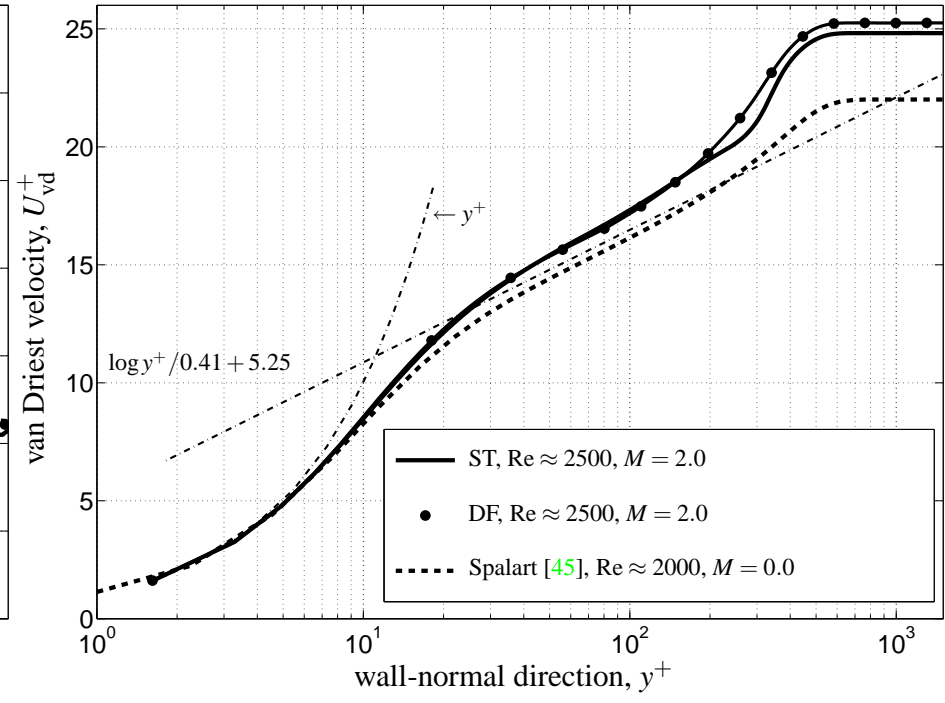

(b) reference van Driest velocity profile

Figure 3: Skin-friction evolution and reference velocity profile: digital filter vs. synthetic turbulence. (The Reynolds numbers quoted are based on the displacement thicknesses obtained from the van Driest-transformed velocity profiles)

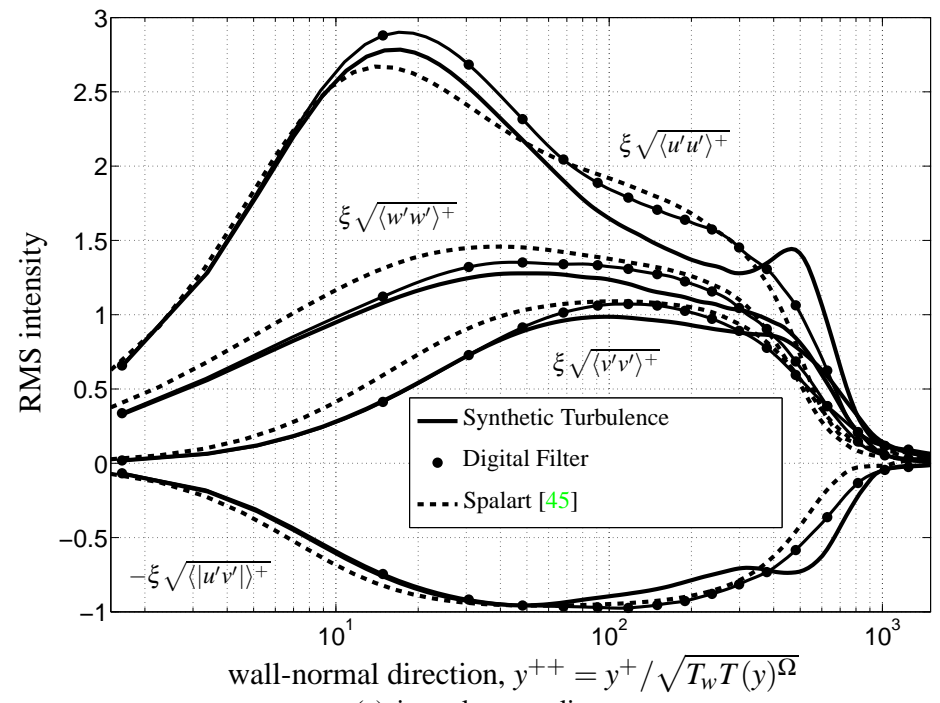

(a) inner-layer scaling

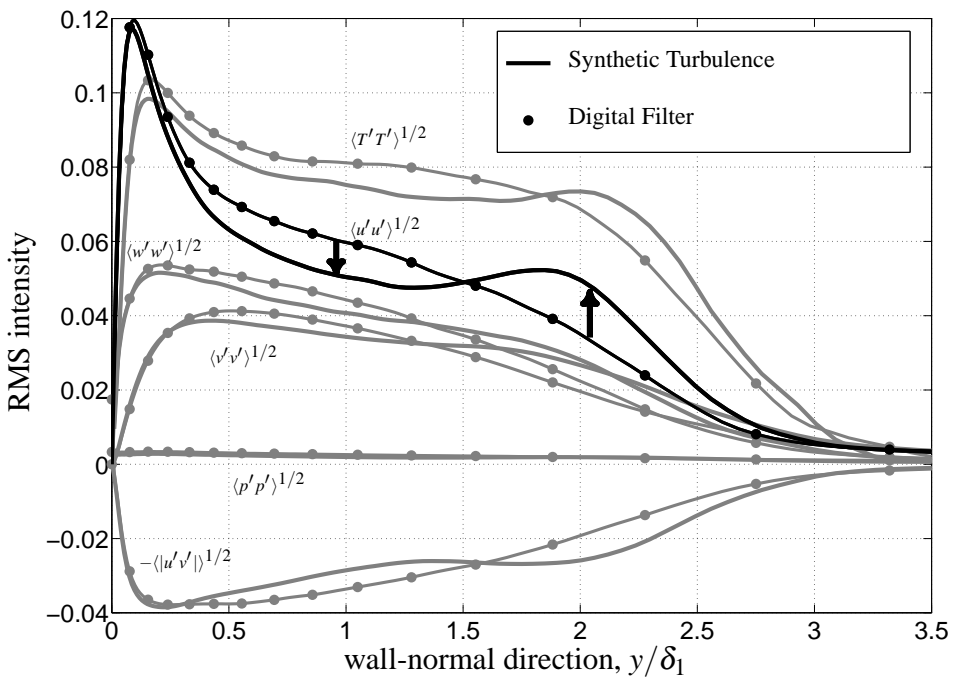

(b) outer-layer scaling

Figure 4: Turbulence intensities: digital filter vs. synthetic turbulence. Note the density correction factor $\xi=\sqrt{\rho(y) / \rho_{w}}$ in $(\mathrm{a})$. The superscript $\Omega$ was taken to be 0.67 . The subscript " w" indicates that the quantity is evaluated at the wall and the prime refers to the quantity fluctuating component

\subsection{Numerical details for the different simulations presented}

For convenience, we provide here the numerical details for all the simulations we will be referring to in the following sections. All of the reported simulations concern the LES of an oblique shock-wave generated by a 8-degree wedge angle impinging on a Mach 2.3 flat-plate-turbulent-boundary-layer at $\operatorname{Re}_{\delta_{1}}$ imp $=21 \times 10^{3}$, consistent with the flow conditions of the IUSTI experiment [11]. Note the use of the superscript "imp" on the displacement thickness. This indicates that the displacement thickness used to compute the Reynolds 
number was evaluated at the nominal impingement location in the absence of the shock during a precursor simulation.

Statistical results involve the grid, domain and subgrid-scale model sensitivity studies. For the grid sensitivity study, we have successively doubled the number of grid points in the three directions of the reference grid (see table 3 for details). Note that the reference grid is similar to the one used by Garnier et al. [19] except in the wall-normal direction where we chose to truncate the problem closer to the wall, at about four boundary-layer thicknesses, in order to reduce the number of grid points used. We thus rely on the ability of the integrated characteristic boundary condition at the top boundary to significantly reduce most of unwanted reflections and preserve the quality of the simulation in the interaction. The domain sensitivity study was performed only in the spanwise direction (see table 4) - this is believed to be the most sensitive domain length. The subgrid-scale model effect was investigated by comparing the MTS model results against the results from the dynamic Smagorinsky model and an implicit LES (see table 4 for details).

Table 3: Numerical details for the grid sensitivity study

\begin{tabular}{|c|c|c|c|c|}
\hline Case & Reference Grid & Refined in $x$ & Refined in $y$ & Refined in $z$ \\
\hline \multicolumn{5}{|l|}{ Domain size } \\
\hline$L_{x} \times L_{y} \times L_{z}$ in $\delta_{1}^{\mathrm{vd}}$ & $450 \times 70 \times 24$ & $450 \times 70 \times 24$ & $450 \times 70 \times 24$ & $450 \times 70 \times 24$ \\
\hline$L_{x} \times L_{y} \times L_{z}$ in $\delta_{1}^{\mathrm{imp}}$ & $71.9 \times 11.2 \times 3.8$ & $71.9 \times 11.2 \times 3.8$ & $70.0 \times 10.7 \times 3.6$ & $72.3 \times 11.3 \times 3.8$ \\
\hline$L_{x} \times L_{y} \times L_{z}$ in $\delta_{0}$ & $25.4 \times 4.0 \times 1.4$ & $25.5 \times 4.0 \times 1.4$ & $25.4 \times 4.0 \times 1.4$ & $25.4 \times 4.0 \times 1.4$ \\
\hline \multicolumn{5}{|l|}{ Number of points } \\
\hline$N_{x} \times N_{y} \times N_{z}$ & $451 \times 81 \times 73$ & $901 \times 81 \times 73$ & $451 \times 161 \times 73$ & $451 \times 81 \times 145$ \\
\hline \multicolumn{5}{|l|}{ Grid resolution $^{\mathrm{a}}$} \\
\hline$\Delta x^{+} \times \Delta y_{\min }^{+} \times \Delta z^{+}$ & $40.6 \times 1.6 \times 13.5$ & $20.3 \times 1.6 \times 13.5$ & $40.6 \times 1.5 \times 13.5$ & $40.6 \times 1.6 \times 6.8$ \\
\hline Grid stretching $^{\mathrm{b}} \beta_{y}$ & 5.50 & 5.50 & $4.75^{\mathrm{c}}$ & 5.50 \\
\hline Time step $\Delta t U_{\infty} / \delta_{1}^{\mathrm{vd}}$ & 0.025 & 0.045 & 0.045 & 0.045 \\
\hline $\begin{array}{l}\text { Statistics acquisition } \\
\text { sampling rate } \\
\text { number of } \text { FTT }^{\mathrm{d}}\end{array}$ & $\begin{array}{c}1 \text { every } 5 \text { steps } \\
9\end{array}$ & $\begin{array}{c}1 \text { every } 5 \text { steps } \\
6\end{array}$ & $\begin{array}{c}1 \text { every } 5 \text { steps } \\
11\end{array}$ & $\begin{array}{c}1 \text { every } 5 \text { steps } \\
7\end{array}$ \\
\hline SGS model & MTS & MTS & MTS & MTS \\
\hline $\begin{array}{l}{ }^{\mathrm{a}} \text { measured upstream } \\
\mathrm{b} \text { the stretching funct } \\
{ }^{\mathrm{c}} \text { note that we chose } \\
\text { layer resolution to b } \\
{ }^{\mathrm{d}} \text { Flow-Through-Tim }\end{array}$ & $\begin{array}{l}\text { of the interaction an } \\
\text { on used is: } y=L_{y} \text { si } \\
\text { keep the same nea } \\
\text { etter capture the sho } \\
: \text { time it takes to go }\end{array}$ & $\begin{array}{l}\text { at the wall for the } \\
\mathrm{h}\left(\beta_{y}(j-1) /\left(N_{y}-\right.\right. \\
\text {-wall resolution as } \\
\text { ks } \\
\text { across the computat }\end{array}$ & $\begin{array}{l}\text { vall-normal directior } \\
) / \sinh \left(\beta_{y}\right) \\
\text { he reference grid an } \\
\text { onal domain at the }\end{array}$ & $\begin{array}{l}\text { increase the outer- } \\
\text { apstream freestream }\end{array}$ \\
\hline
\end{tabular}

In the later sections of the paper, we consider the SBLI dynamics. The integration times covered by the above simulations were found to be insufficient to investigate the low-frequency content, if any, of the interaction. To overcome this issue, the small-span LES was continued for an extended amount of time as it is clearly the cheapest case we could run. For that reason, our results on the unsteady aspects of the interaction were obtained from the time-integration of the small-span LES over 4 million iterations, after a start-up transient of half a million iterations. This represents about 408 flow-through-times. Using the experimental value of $115 \delta_{0} / U_{\infty}$ for the period of the most energetic low-frequency oscillation, the current LES signal should cover about 90 cycles at such frequency. This makes a Fourier analysis possible at low-frequencies, which was missing in previous studies of this kind.

Table 4: Numerical details for the domain and SGS model sensitivity study

\begin{tabular}{|c|c|c|c|c|}
\hline Case & Large span $\left(5 L_{z}\right)$ & Small span $\left(L_{z} / 2\right)$ & Dyn. Smagorinsky & Implicit \\
\hline \multicolumn{5}{|l|}{ Domain size } \\
\hline$L_{x} \times L_{y} \times L_{z}$ in $\delta_{1}^{\mathrm{vd}}$ & $450 \times 70 \times 120$ & $450 \times 70 \times 12$ & $450 \times 70 \times 24$ & $450 \times 70 \times 24$ \\
\hline \multicolumn{5}{|l|}{ Number of points } \\
\hline$N_{x} \times N_{y} \times N_{z}$ & $451 \times 81 \times 361$ & $451 \times 81 \times 37$ & $451 \times 81 \times 73$ & $451 \times 81 \times 73$ \\
\hline Grid stretching $\beta_{y}$ & 5.50 & 5.50 & 5.50 & 5.50 \\
\hline Time step $\Delta t U_{\infty} / \delta_{1}^{\mathrm{vd}}$ & 0.045 & 0.045 & 0.045 & 0.045 \\
\hline $\begin{array}{l}\text { Statistics acquisition } \\
\text { sampling rate } \\
\text { number of } \mathrm{FTT}^{\mathrm{a}}\end{array}$ & $\begin{array}{c}1 \text { every } 5 \text { steps } \\
9\end{array}$ & $\begin{array}{c}1 \text { every } 5 \text { steps } \\
73\end{array}$ & $\begin{array}{c}1 \text { every } 5 \text { steps } \\
16\end{array}$ & $\begin{array}{c}1 \text { every } 5 \text { steps } \\
8\end{array}$ \\
\hline SGS model & MTS $^{b}$ & MTS $^{b}$ & Dyn. Smagorinskyb & None $^{b}$ \\
\hline
\end{tabular}

${ }^{a}$ Flow-Through-Time: time it takes to go across the computational domain at the upstream freestream velocity

${ }^{\mathrm{b}} \mathrm{a} 6^{\text {th }}$-order filter was applied every 5 iterations to remove spurious numerical oscillations 


\section{Time-averaged fields and flow statistics}

\subsection{Comparison with the PIV data}

In this section, we compare the flow statistics obtained from the large-span LES run against the available PIV data [12] of the same flow. Figure 5 gives the time-averaged streamwise-velocity field. The left-hand side of the figure is a superposition of the PIV field (in filled contours) and the LES field (thick solid lines). The contours were taken at exactly the same levels to allow a direct comparison of both the spatial structure and amplitude level of the velocity fields. The right-hand side of the figure provides a comparison of the PIV and LES velocity profiles at four different streamwise locations. Overall, the LES results are in good agreement with the PIV data. One noticeable difference is in the separation area (highlighted in the contourmaps) where the PIV finds a taller mean separation bubble with a slightly stronger reserved flow (about $5 \%$ of the upstream freestream velocity in the PIV against 3\% in the LES). The boundary-layer thickening, however, is well captured.
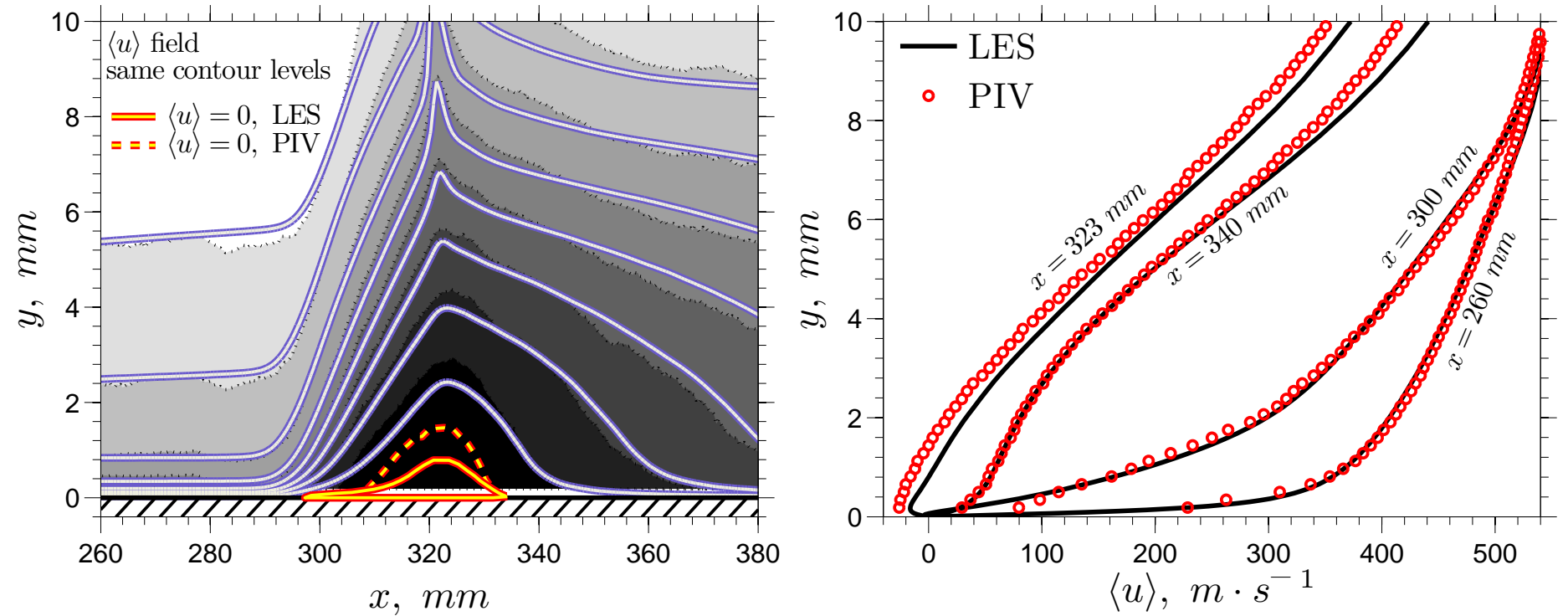

Figure 5: Mean streamwise velocity: PIV vs. LES. Two-dimensional distribution showing the PIV in filled contours and the LES in solid lines at exactly the same contour levels (left). Profiles at different streamwise locations (right)

Figure 6 is a similar comparison as in the previous paragraph but for the wall-normal velocity component. The initial part of the interaction is in good agreement with the PIV. In the recovery, the agreement near the wall is satisfactory, but deteriorates in the outer part of the boundary layer. Larger differences are seen in the separation bubble, as mentioned in the above paragraph. It should be noted that the PIV is less converged for the wall-normal velocity than for the streamwise velocity and that near-wall PIV measurements are usually less reliable. Furthermore, the flow inside the bubble is very unsteady and the bubble can be nonexistent at times and much bigger than its mean size at other times. The velocity fluctuations inside the bubble can thus be large compared to the mean velocity value, producing high Reynolds-stress values. Therefore, we do not expect a very good agreement between the LES and the PIV inside the mean separation.

Figure 7 gives the map of the root-mean-square (RMS) of the streamwise velocity fluctuations. The LES results are seen to capture a structure similar to the PIV one inside the interaction. In particular, the inclination angle of the high-intensity ridge found inside the interaction region is in good agreement with the PIV findings. The ridge corresponds to the energetic shear layer formed at the bubble interface. It can be seen, however, that the shear layer in the LES is slightly thinner than in the PIV. Also, it should be noted that the fluctuations in the LES upstream of interaction do not penetrate as far into the flow as in the PIV. This is due to the digital-filter settings, which assigned too few energy in the outer part of the boundary layer. This was improved in a more recent simulation, which we do not report here. However, the lack of incoming outer-layer fluctuations does not seem to survive past the interaction and the profiles at $x=340 \mathrm{~mm}$ are in good agreement.

In figure 8, we report the comparison of the wall-normal velocity fluctuations. It can be seen that the comparison deteriorates compared to the previous figures: the LES fluctuations are a bit stronger in the post-interaction region and the local maximum seems to be closer to the wall than in the PIV. The shift in the height of the ridge of maximum wall-normal velocity fluctuations seems to correlate well with the taller PIV bubble. It is unclear why the experimental bubble is taller, but one can speculate this to be related to the presence of the wind-tunnel side walls, which tend to enhance the size of the separation bubble ${ }^{3}$. However, note that the contourmap indicates a good match for the shock-system position, suggesting that the size of the interaction found by the LES is in good agreement with the experiment.

Finally, figure 9 gives the Reynolds shear-stress distributions. It must be emphasized that the shear stress is not easily obtained using PIV. Nevertheless, despite the lack of convergence in the PIV data, the qualitative and to some extent the quantitative agreement between the PIV and the LES is remarkably good. It is interesting to note the small but clear region of high positive shear-stress values (near $x=320 \mathrm{~mm}$ and $y \in[7 \mathrm{~mm}, 10 \mathrm{~mm}]$ ). This corresponds to the flapping motion of the incident-shock tip. Also, one can detect the

${ }^{3}$ Private communication with Dr. Jean-Paul Dussauge, IUSTI (2008) 

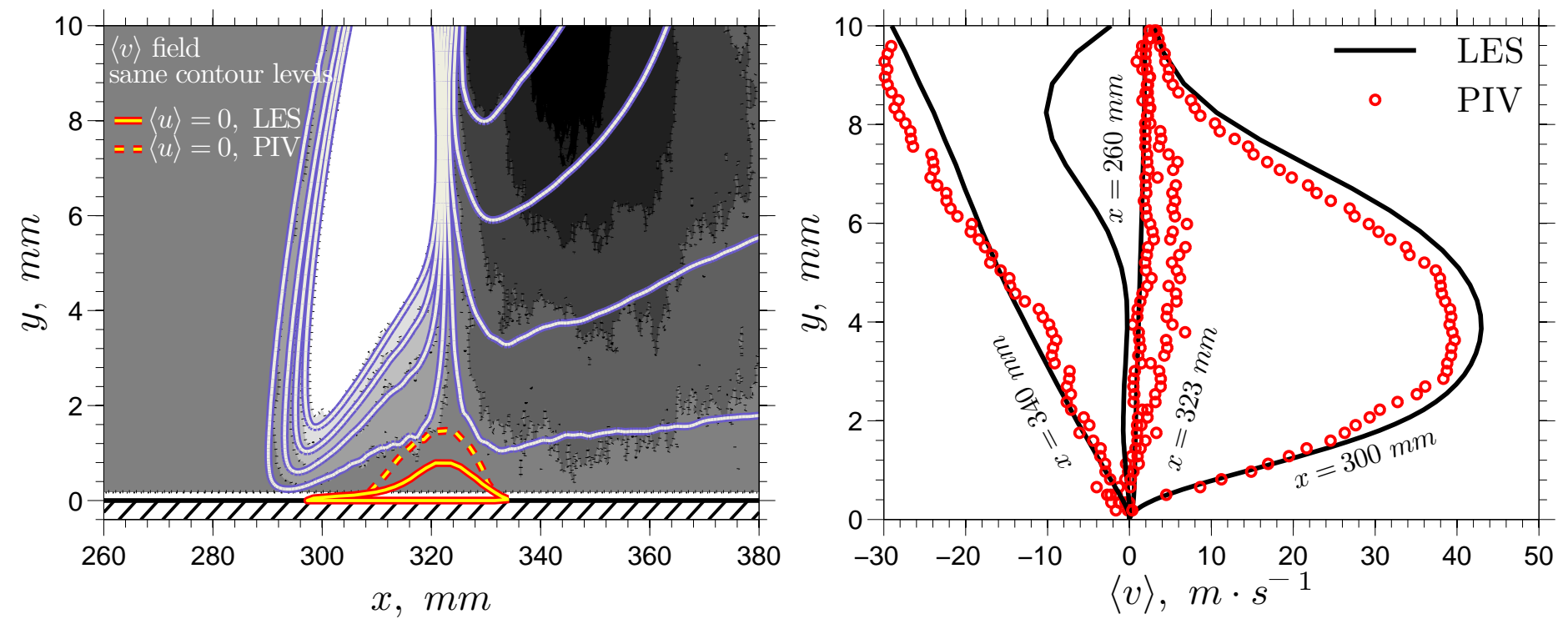

Figure 6: Mean wall-normal velocity: PIV vs. LES. Two-dimensional distribution showing the PIV in filled contours and the LES in solid lines at exactly the same contour levels (left). Profiles at different streamwise locations (right)
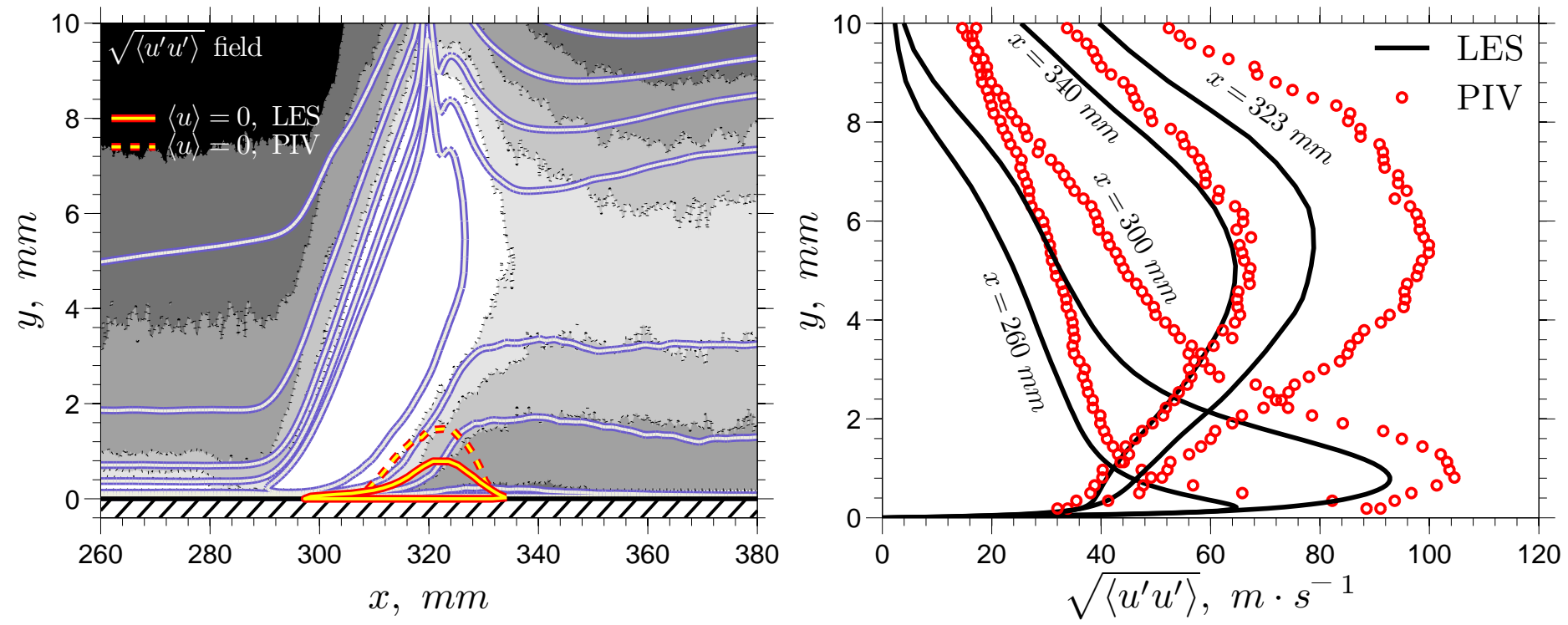

Figure 7: RMS of the streamwise velocity fluctuations: PIV vs. LES. Two-dimensional distribution showing the PIV in filled contours and the LES in solid lines at exactly the same contour levels (left). Profiles at different streamwise locations (right)

mean position of the reflected shock, which is seen to be correctly predicted by the LES.

Generally speaking, the PIV and LES data agree sufficiently well to believe in the ability of LES to reproduce this complex flow field. Also, the good agreement with the PIV data, taken from the median plane of the wind tunnel, suggests that the 8-degree-wedge-angle experiment is close to being statistically two-dimensional, as claimed by Dussauge and Piponniau [14], and that the wind-tunnel-corner flows are not too important in this case. However, it was clearly shown in this section that the separation-bubble predictions were good for the bubble length but that the bubble height was underpredicted by the LES. This is believed to be the sign of some level of three-dimensionality in the experiment but we emphasize that the interaction-length prediction would not be in good agreement if the experiment had been strongly affected by the side walls, as shown for example in the 9.5-degree case [14]. In the 9.5-degree case, the success of a statistically two-dimensional LES would not be guaranteed and would probably have to account for the wind-tunnel side-wall effects. ${ }^{4}$

\subsection{Sensitivity of the results to the grid resolution, domain width and choice of subgrid-scale model}

In this section, we look at the sensitivity of the base flow to the choice of grid, domain width and SGS model. Figure 10(a) gives the skin friction evolution inside the interaction for different grid resolutions, as defined in table 3. Although the statistics were not acquired over the same amount of samples, the number of samples used in this study was large enough to consider the results to be statistically converged. From figure 10(a), we do not find the tested grid resolution to produce significant differences in the size of the separation bubble and skin-friction levels. Garnier et al. [19] also looked at the sensitivity of their results to the grid resolution and could not find

\footnotetext{
${ }^{4}$ Private communication with Dr. Eric Garnier, ONERA (2007)
} 

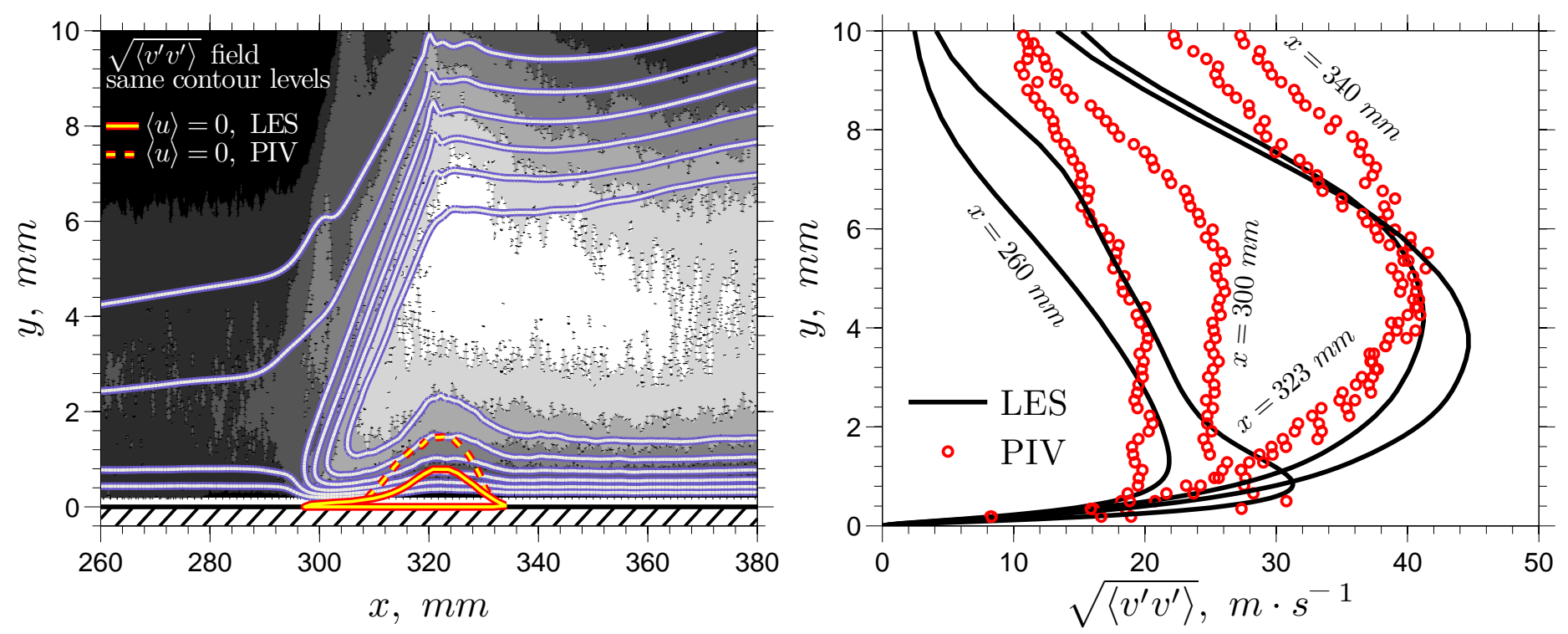

Figure 8: RMS of the wall-normal velocity fluctuations: PIV vs. LES. Two-dimensional distribution showing the PIV in filled contours and the LES in solid lines at exactly the same contour levels (left). Profiles at different streamwise locations (right)
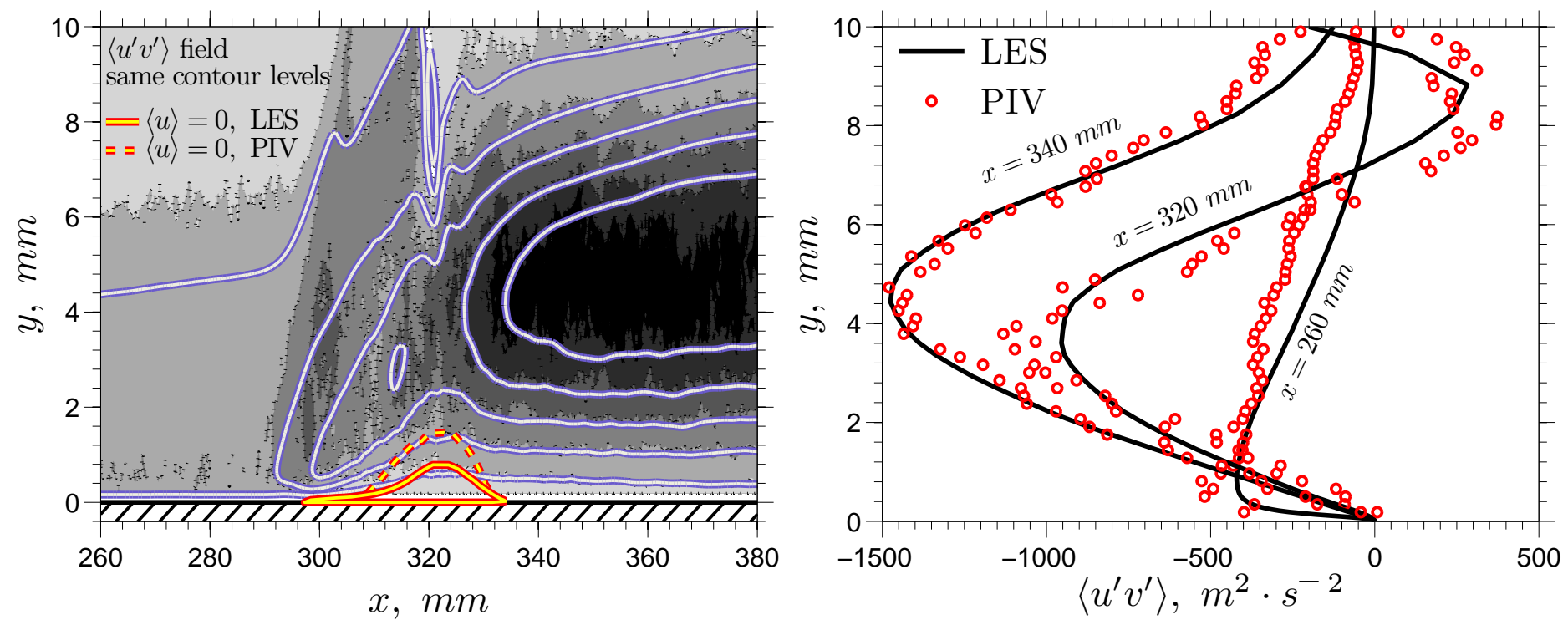

Figure 9: Reynolds shear stress: PIV vs. LES. Two-dimensional distribution showing the PIV in filled contours and the LES in solid lines at exactly the same contour levels (left). Profiles at different streamwise locations (right)

any significant differences at similar resolutions to the present ones (note that they used the local boundary-layer-edge conditions to normalize the skin friction and Sutherland's law for the dynamic viscosity while we use the upstream boundary-layer-edge conditions and a power law). Thus, we are confident that the grid resolution used is sufficiently fine to only have marginal effects on the statistical results.

Figure 10(b) compares the skin-friction evolution for the different domain widths considered (see table 4). Contrary to the grid sensitivity tests, we find the results to be very sensitive to the computational-box width. Previous simulations of the IUSTI 8-degree shock-reflection case made use of spanwise widths of $1.4 \delta_{0}$ in Garnier et al.[19] and $2.2 \delta_{0}$ in Pirozzoli and Grasso [37], while the experimental separation-bubble length is $O\left(4 \delta_{0}\right)$-long [11]. In this domain-size effect study, we have tested spanwise lengths ranging from $0.7 \delta_{0}$ to $7 \delta_{0}$. The separation point is found to move upstream as we reduce the spanwise extent of the domain while the reattachment point moves further downstream, leading to larger bubbles and slower recovery rates. Figure 11(a) further confirms the changes by looking at the wall-pressure distribution (normalized by the upstream pressure). The wall-pressure distribution is seen to develop a plateau as the domain width is reduced. This is reminiscent of laminar interactions, but we are certain that the flow remained fully turbulent in the small-span simulations. In fact, the small-span LES was chosen such that despite the increased spanwise coherence (forced by the periodic boundary conditions) we would still maintain a fully turbulent boundary layer. The existence of a pressure plateau is thus a direct consequence of the bubble extension due to the high level of spanwise coherence. Finally, it is interesting to note that the increased interaction length due to the reduced size of the domain width does not seem to affect the initial rate of change of the wall-pressure distribution. This is reminiscent of the free-interaction theory in laminar interactions [46, 28].

Table 5 shows the bubble and interaction lengths for the different grids and domain sizes, compared with the values obtained by Garnier et al. [19] (LES) and Dupont et al. [11] (experiment). This further quantifies the sensitivity of the bubble to the domain width, 


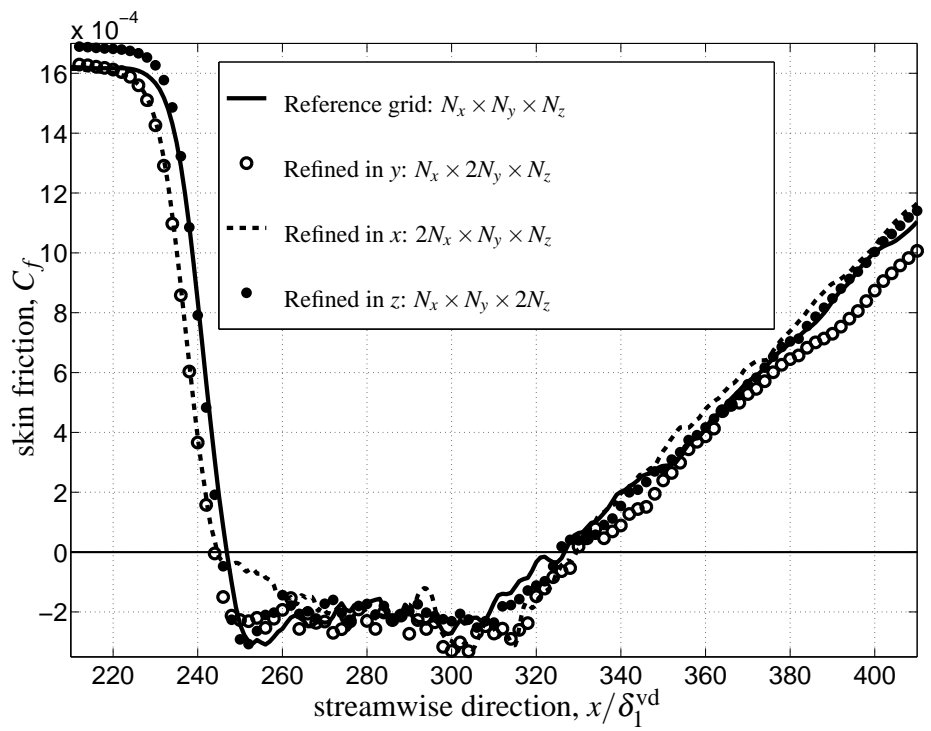

(a) grid-resolution effect

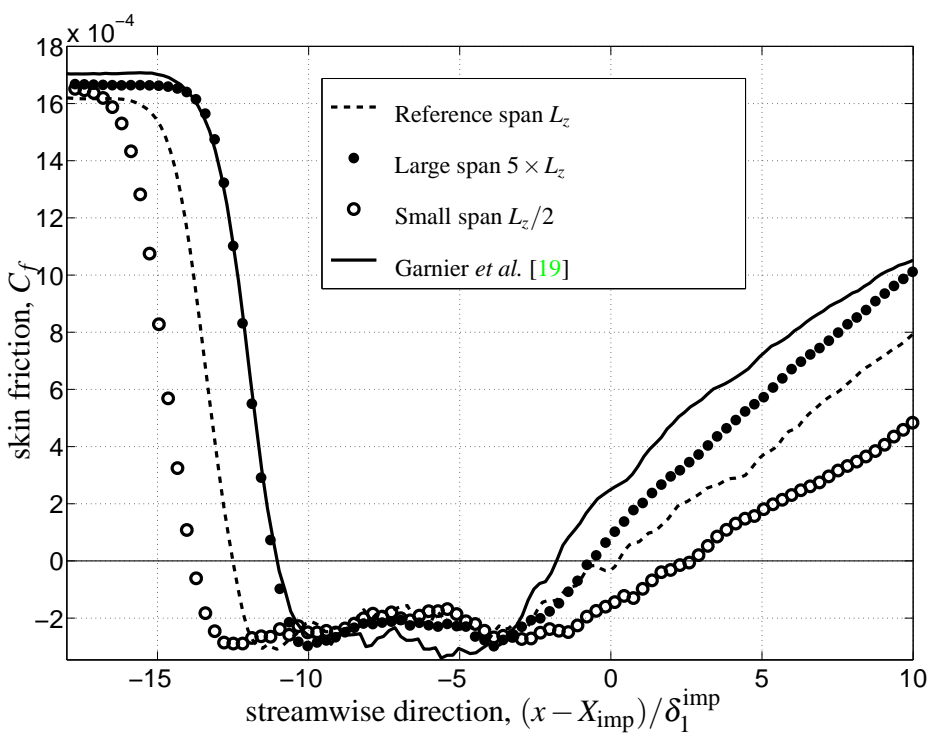

(b) domain-width effect

Figure 10: Skin-friction sensitivity to the grid resolution and the domain width. The skin friction is normalized with the upstream potential-flow properties and the dynamic viscosity is computed using the power law. $X_{\text {imp }}$ is the inviscid-impingement location of the incident oblique shock and $\delta_{1}^{\mathrm{imp}}$ is the boundary-layer displacement thickness at $X_{\mathrm{imp}}$ in the absence of the shock

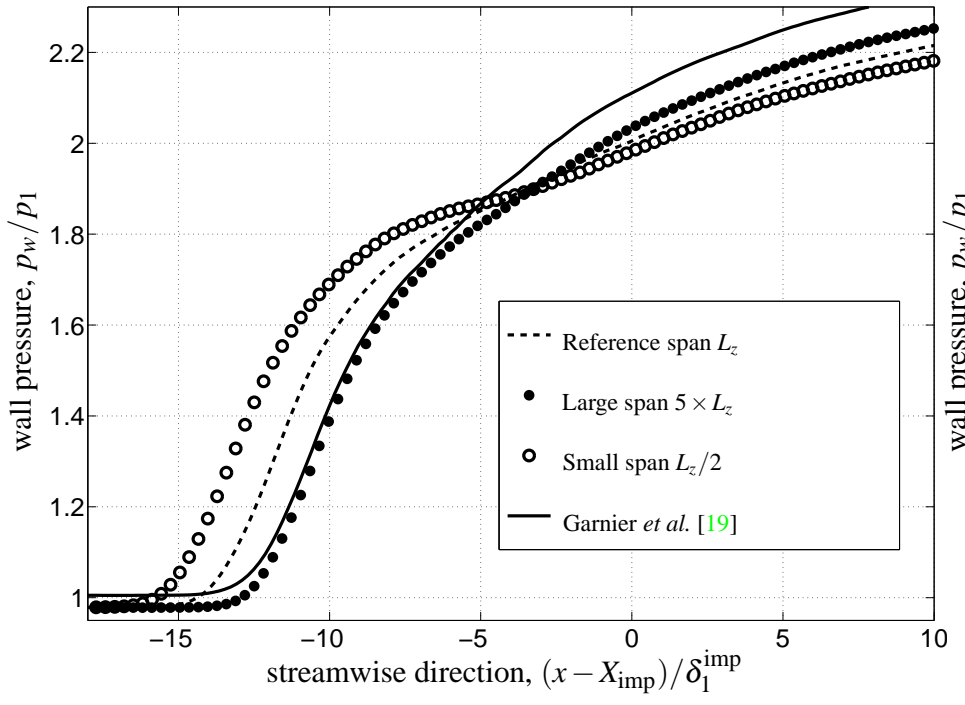

(a) domain-width effect

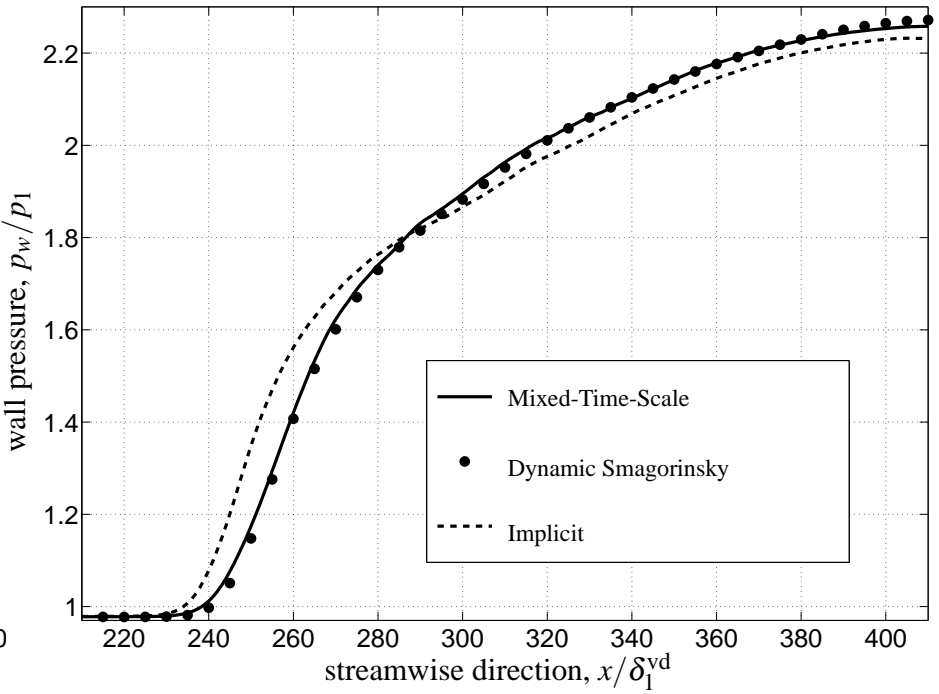

(b) subgrid-scale model effect

Figure 11: Wall pressure sensitivity to the domain width and the subgrid-scale model. The pressure $p_{1}$ is the theoretical freestream pressure before the incident shock wave

with an extension of the bubble of about $35 \%$ between the large and small-span cases. In addition, table 5 suggests that the simulated normalized shock intensity is higher than in the experiment, probably due to a slightly lower level in the incoming skin friction. In the table, $p_{2}$ refers to the theoretical freestream pressure after the incident shock but before the reflected shock and $\tau_{w}$ is the wall shear-stress before the interaction. Finally, table 5 quantifies the differences found between the interaction length $L$ and the separation length $L_{\text {sep }}$. The interaction length is defined as the distance between the location of the reflected-shock extension to the wall $X_{0}$ and the inviscid-impingement location of the incident oblique shock $X_{\mathrm{imp}}$ while the separation length is the distance between the separation point $X_{\text {sep }}$ and the reattachment point $X_{\text {rea. }}$. Experimentalists prefer to use the interaction length while computationalists favor the use of the separation length, which is evaluated more precisely. In the remaining part of the paper, we will use the separation length rather than the interaction length. To allow a consistent comparison with the experiment, we have evaluated the experimental separation length (based on the LES results) to be around $39 \mathrm{~mm}$. This will be important for the unsteady aspects.

The subgrid-scale-model effect was investigated by comparison of the MTS model with the dynamic Smagorinsky model and an implicit LES approach. Figure 11(b) gives the wall-pressure rise in the interaction obtained from the different models. We find that the MTS and the dynamic Smagorinsky (DS) models give very similar results, while the implicit LES stands out. The good agreement between the two models suggests that the grid is fine enough so that the particular choice of eddy-viscosity model has little importance. However, the larger separation found by the implicit LES, as shown in figure 12(a), and steeper increase in the 
Table 5: Interaction lengths and normalized shock intensity

\begin{tabular}{lcccccccc}
\hline \hline Case & Ref. & $2 N_{x}$ & $2 N_{y}$ & $2 N_{z}$ & $L_{z} / 2$ & $5 L_{z}$ & Garnier et al. [19] & Dupont et al. [11] \\
\hline$\left(p_{2}-p_{1}\right) / 2 \tau_{w}$ & 50.2 & 50.9 & 50.5 & 48.6 & 49.3 & 48.9 & 47.5 & 40.5 \\
\hline$L / \delta_{0}$ & 5.1 & 5.4 & 5.3 & 5.1 & 5.9 & 4.8 & 4.5 & 4.2 \\
$L_{\text {sep }} / \delta_{0}$ & 4.5 & 4.8 & 4.8 & 4.5 & 6.1 & 3.9 & 3.1 & $3.5^{\mathrm{a}}$ \\
\hline$\left(X_{\text {sep }}-X_{0}\right) / \delta_{1}^{\text {imp }}$ & 2.0 & 2.4 & 2.1 & 1.7 & 2.1 & 2.0 & 2.5 & - \\
$\left(X_{\text {rea }}-X_{\text {imp }}\right) / \delta_{1}^{\text {imp }}$ & 0.2 & 0.7 & 0.7 & 0.0 & 2.7 & -0.7 & -1.8 & - \\
\hline \hline
\end{tabular}

a this value is not in the original paper of Dupont et al. [11] but was estimated based on the LES results taking $\left(X_{\text {sep }}-X_{0}\right) / \delta_{1}^{\text {imp }} \sim 2$, giving an experimental value of $L_{\text {sep }} \approx 39 \mathrm{~mm}$

wall-pressure (figure 11(b)) leads us to believe that simply neglecting the SGS terms for that particular grid would not be correct.

In figure 12(b) we look at the SGS model effect on the incoming velocity profile. The DS model gives a slightly lower friction velocity than the MTS and implicit LES, as already noticed in the upstream skin-friction values. The apparent overshoot of the log-law constant is not believed to be related to a resolution issue, since the grid-refinement study did not show any strong deviations in the results as the grid was refined. In fact, the overshoot is mainly due to the choice of dynamic-viscosity law. In the present LES, we use the power law with exponent 0.67. If instead, we use Sutherland's law (as in Garnier et al. [19]), it can easily be shown that the dynamic viscosity at the wall would be about $13 \%$ greater. To estimate the effect of a $13 \%$ difference in the dynamic-viscosity value at the wall, the van Driest velocity profile from the MTS model was re-processed using Sutherland's law and the result is shown in figure 12(b). The difference is clear and the agreement with the log-law is much better. Furthermore, it must be noted that there exist some variations on the value of the additive constant used in the literature (van Driest [51] used 5.24).

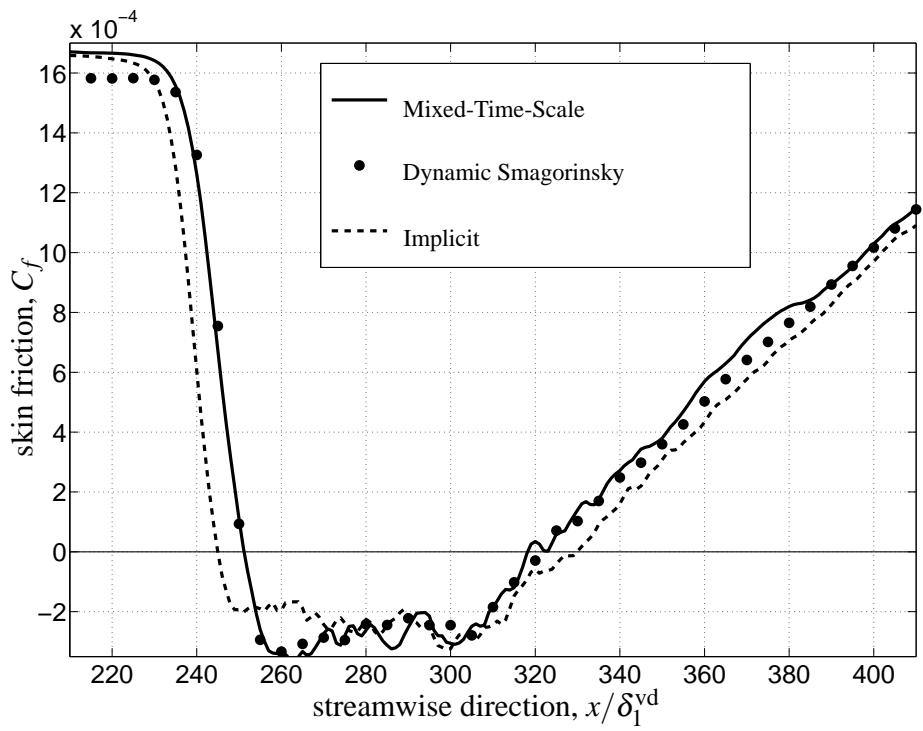

(a) subgrid-scale model effect on the skin friction

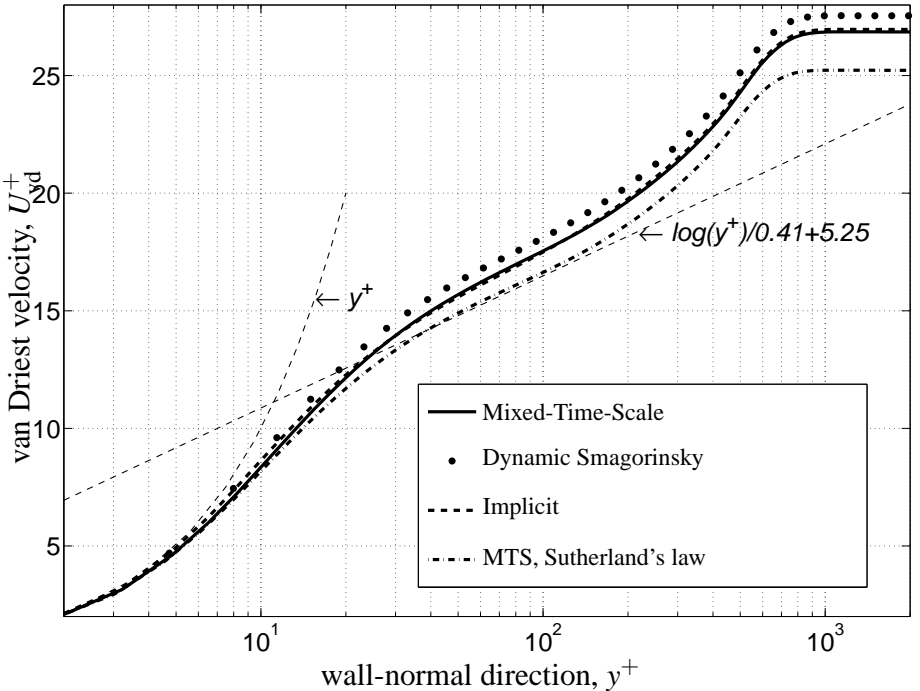

(b) subgrid-scale model effect on the upstream velocity profile

Figure 12: Subgrid-scale model effect on the upstream velocity and the interaction region

Finally, figure 13 gives the eddy-viscosity field for the two SGS models tested. The asymptotic behavior of the models in the upstream boundary layer as we approach the wall differ, as shown in figure 13(a). The eddy viscosity from the MTS model approaches the wall as $y^{+^{2}}$, which is a factor $y^{+}$away from the expected asymptotic behavior, properly captured by the DS model. This issue is reported in Inagaki et al. [27]. Despite the wrong near-wall behavior of the MTS model, the skin friction in the relaxation part of the interaction (see figure 12(a)) is close to the one from DS, suggesting that the incorrect asymptotic behavior of the MTS model has little importance. Figure 13(b) confirms the overall similar eddy-viscosity distribution between the two models inside the interaction, with some discrepancies near the shocks.

Since the DS model is significantly more computationally intensive than the MTS model, for no obvious additional improvements in the SBLI predictions, and that the implicit-LES results differ from the ones with the SGS model on, the choice of the MTS model in the present SBLI studies appears justified.

\section{Linear stability study}

Before presenting the unsteady aspects of the LES data, we consider in this section the stability properties of the time-averaged flow. As mentioned in the introduction, Robinet [40] has recently performed a Bi-Global analysis of an oblique shock impinging on a laminar boundary layer, and found that for a sufficiently large wedge angle, the flow could become absolutely unstable to spanwise wavelengths of the order of the separation-bubble length. The question we intend to answer here is whether or not the time-averaged flow of the 


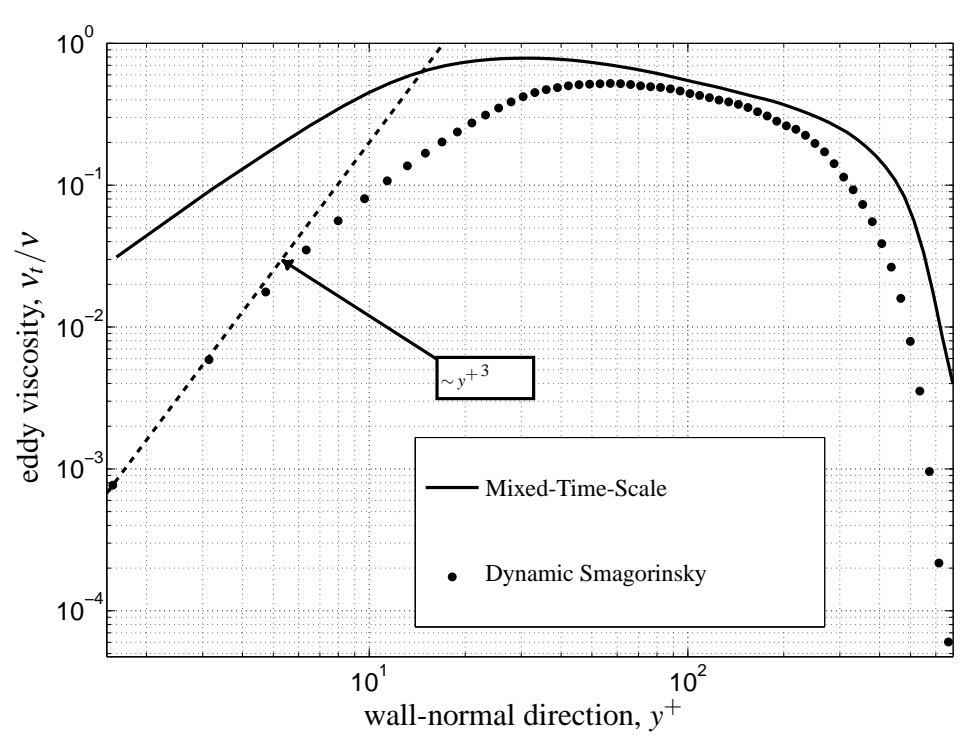

(a) model effect on the upstream eddy-viscosity profile

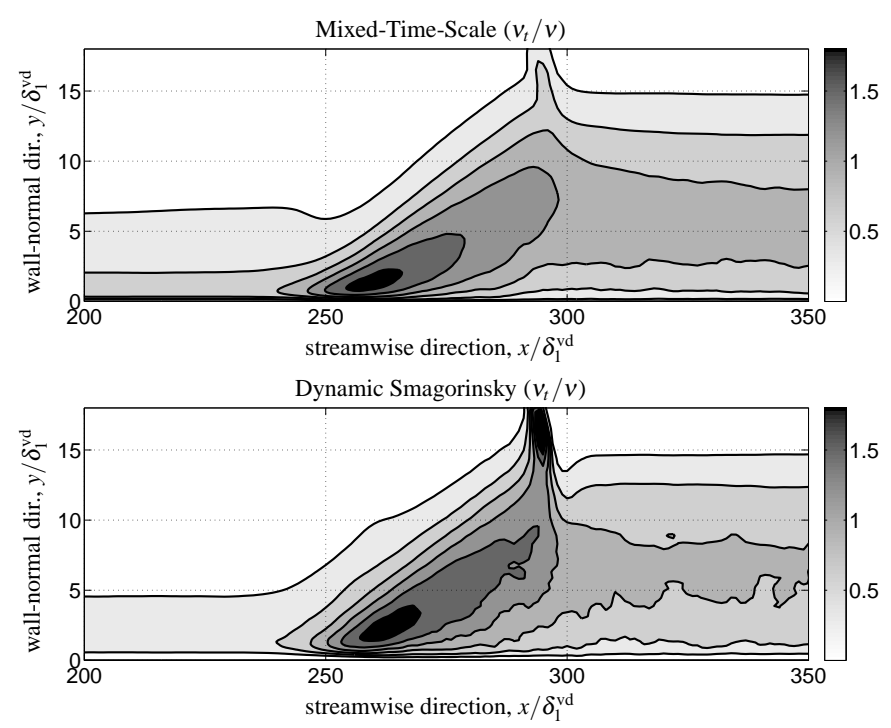

(b) model effect on the eddy-viscosity distribution

Figure 13: Subgrid-scale model effect on the eddy viscosity to kinematic viscosity ratio

current turbulent interaction has a similar property. To address this issue, we chose to use the SBLI code but modified to detect the most unstable or least damped mode of any given (3D) base flow.

\subsection{Method}

As mentioned earlier in the paper, the in-house code solves the 3D compressible Navier-Stokes equations, which we re-write in the following generic way:

$$
\frac{\partial q}{\partial t}=\operatorname{RHS}(q)
$$

where $q$ is the conservative variable vector $\left[\rho, \rho u, \rho v, \rho w, \rho E_{t}\right]^{\mathbf{T}}$. Let us denote by $q_{b}$ the time and span-averaged field from the LES results. In this section, we will refer to $q_{b}$ as the base flow. If we use this base flow as the initial condition of the SBLI code, we can obtain the rate of change of $q$ needed to satisfy the equilibrium. In other words:

$$
\frac{\partial q_{b}}{\partial t}=\operatorname{RHS}\left(q_{b}\right)
$$

If we now decompose the flow $q$ into the base-flow component $q_{b}$ and a perturbation $q^{\prime}\left(q=q_{b}+q^{\prime}\right)$, we can write:

$$
\frac{\partial q^{\prime}}{\partial t}=\operatorname{RHS}(q)-\operatorname{RHS}\left(q_{b}\right)
$$

where RHS $\left(q_{b}\right)$ acts like a forcing term in the governing equations. This allows us to look at the time and spatial evolution of a prescribed initial disturbance on the base flow while maintaining the base flow at its initial state. Thus, we can detect the most unstable (or least damped) mode. The above forcing was introduced in the SBLI code with no inclusion of the SGS terms to run the stability analysis as a DNS. However, the TVD-Ducros filter and the integrated characteristic schemes were used and modified in a similar way to the governing equations given above.

The base flow is the time-averaged flowfield and is not a solution to the Navier-Stokes equations, overwise the left-hand side of equation (20) would strictly be zero. In fact, the forcing term introduced can be thought of as the divergence of a Reynolds-stress tensor in the RANS equations. Thus, the above stability-analysis formulation is similar to the linear-stability analysis of the RANS equations, if we consider small perturbations. It could then be argued that such analysis corresponds to the initial stage of an unsteady RANS calculation started from the steady-RANS solution, but with the major difference that no modelling is applied to the disturbances. There are cases where the large-scale flow unsteadiness are found to be relatively well predicted by linear theory. For example, Gaster et al.[20] compare their experimental measurements of a forced turbulent mixing layer with the results of classical linear-stability theory, and report a good agreement between the two in both the amplitude and phase distribution. A more recent successful attempt is the application of the BiGlobal analysis to predict the shock-induced transonic-buffet onset by Crouch et al. [7]. It can thus be argued that the SBLI case may also be a candidate for the application of linear-stability theory. Indeed, the low-frequency shock motions are known to occur on timescales two orders of magnitude larger than the characteristic timescale of the turbulence. The separation of timescales, which appears to be needed for a successful extension of linear-stability theory, is clearly present in the SBLI case. In such a framework, the turbulent nature of the flow is thus only needed to produce the time-averaged base flow.

\subsection{Results}

To first check the validity of the above modifications of the code, we have run the stability simulation with no initial disturbances and could maintain the base flow for as long as we have performed the test for (about six flow-through-times, longer than is needed 
for this study). We then introduced white noise disturbances with maximum amplitude of 4,6 or 8 orders of magnitude smaller than the free-stream quantities. The white noise was introduced upstream of the interaction inside a square cylinder of section 5 by 5 grid points spanning the entire box. Alternatively, we have also excited specific spanwise wavenumbers by using sine waves for the initial condition. The linear-stability simulations were performed for various domain widths, ranging from 0 up to 8 separation-bubble lengths. The original LES grid resolution was kept in the streamwise and wall-normal directions whereas the spanwise resolution was set to 20 and 40 points per separation-bubble wavelength (two resolutions were tested to make sure that the results are grid independent). The base flows considered here were obtained by time and span-averaging the conservative variables during the reference, the small, and the large-span LES runs. To remove spurious oscillations in the time and span-averaged data, the base flows were filtered prior running the stability simulations.

After a transient state, all the tested cases have shown that the disturbances end up picking up a globally unstable mode (following an exponential growth in time), the structure of which is shown in figure 14(a). The mode was found to be stationary until saturation of the linear regime was reached.

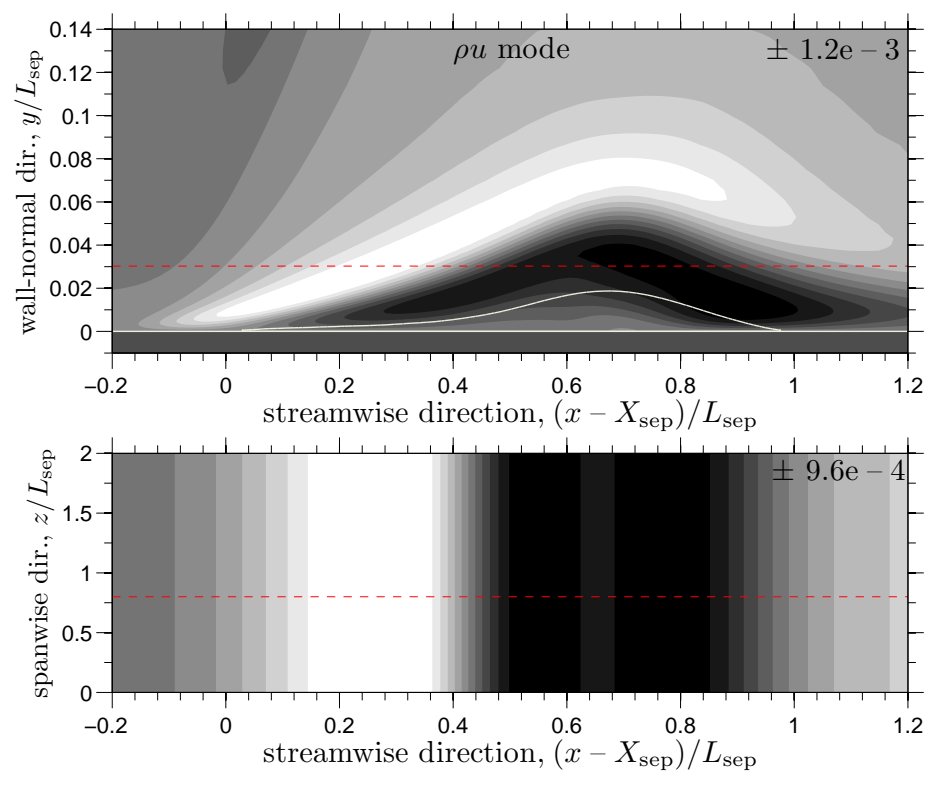

(a)

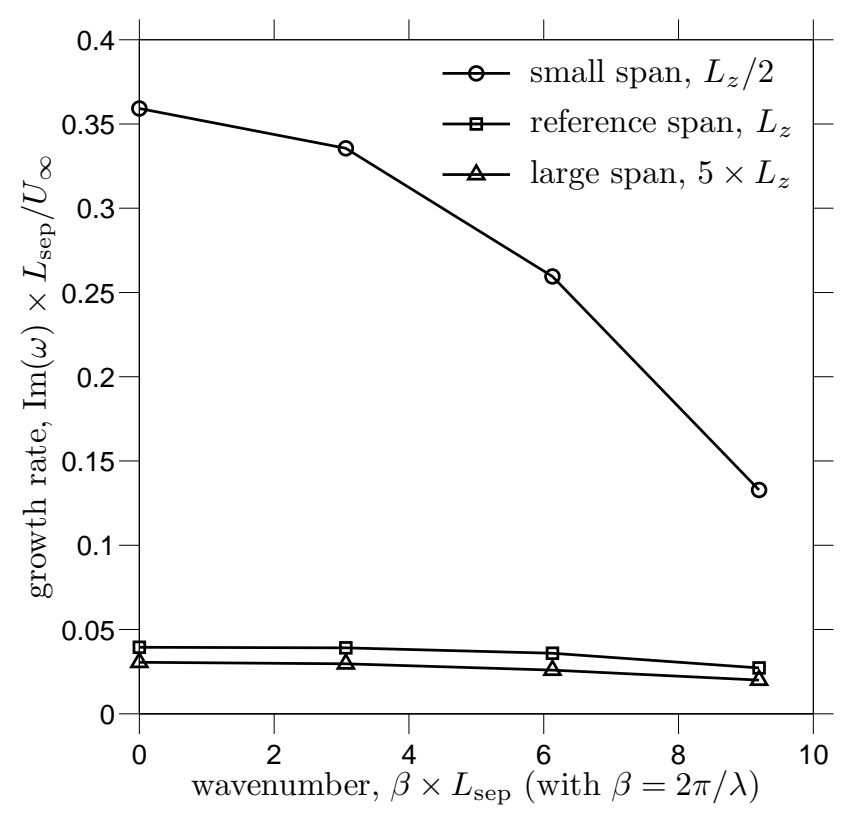

(b)

Figure 14: Existence of an absolutely unstable global mode. (a) Amplitude function (side and top views) of the streamwise momentum disturbance ( $\rho u$-mode), where the white line indicates the base-flow zero-velocity contour. The contours are equally spaced between plus and minus the maximum amplitude (on a logarithmic scale) (b) Growth rates for different spanwise wavenumbers, where $\{\rho u\}^{\prime}=$ $A(x, y) \exp (i \beta z-i \omega t)$, with $A(x, y)$ the amplitude function and $i$ the imaginary number; for the different base flows tested

Contrary to Robinet's [40] results on a laminar interaction, we find the global mode to be present in 2D (i.e. at zero spanwise wavenumber) for the present turbulent SBLI case. Furthermore, when trying to enhance higher wavenumbers in the initial disturbance, we consistently found the smaller wave-numbers to be growing faster. Figure 14(b) provides the growth rates obtained at different wavenumbers from the time evolution of the amplitude of different spanwise-Fourier modes. First, we find the 2D mode to be the most unstable, although 3D modes with wavelengths of the order of, or larger than, the separation-bubble length have very similar growth rates. Second, we find that the growth rates are low compared to the inverse time scales involved in the turbulence. In fact, the growth rates are found to be smaller than an inverse time scale based on the free-stream velocity and the separation length. However, the values provided in figure 14(b) should be considered with care as they are shown to be sensitive to the base flow used. Nevertheless, we can say that if the 2D global mode is active, the associated amplification mechanism would scale on several bubble-flow-through times.

If we now consider the $2 \mathrm{D}$ structure of the global mode, it is worth noting that the sign of the amplitude function is arbitrary. In fact, changing the sign of the initial disturbance leads to the same picture as in figure 14(a) with the difference that the sign of the amplitude function is reversed. The effect of the global-mode structure on the skin friction is given in figure 15 . Depending on the sign of the amplitude function, one can show that the separation and reattachment points are either moved upstream or downstream (in phase). Furthermore, as shown in figure 15(b), the bubble can either break up or the separation can be amplified in the initial portion of the separation under the influence of the global mode (note that the disturbance amplitude levels were increased to a non-linear level to make the global-mode effect visible). The relevance of this remark will be made clearer later in the discussion of the LES data.

We thus have found an unstable global mode in the span- and time-averaged flow field of the turbulent SBLI, the growth rate of which is greatest at zero spanwise wavenumber. Going back to the discussion in section 4.1, it should be noted that the global-mode 


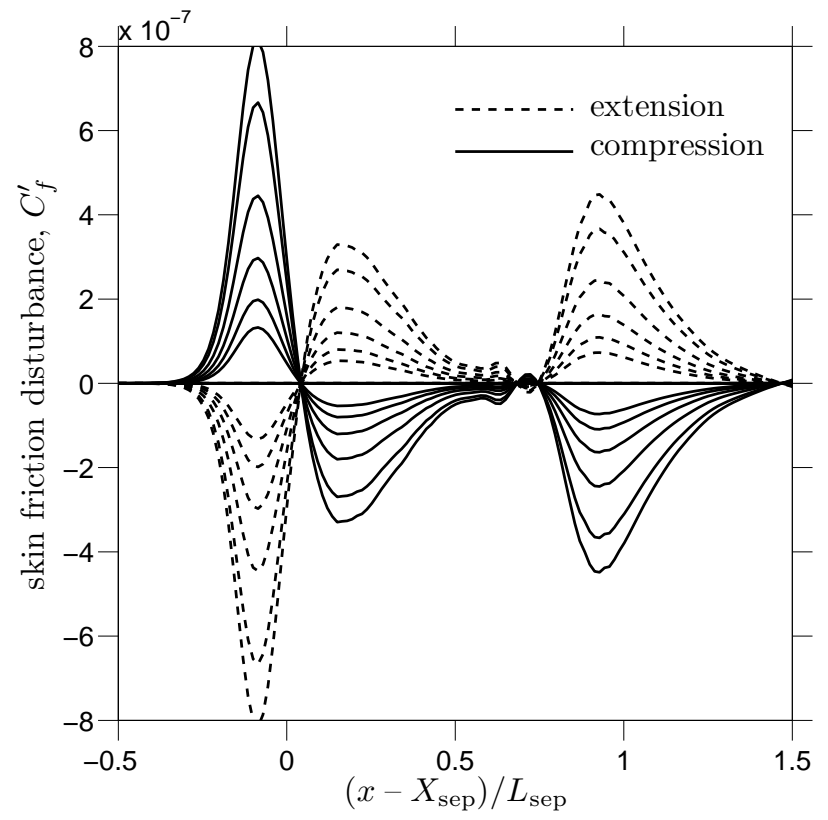

(a)

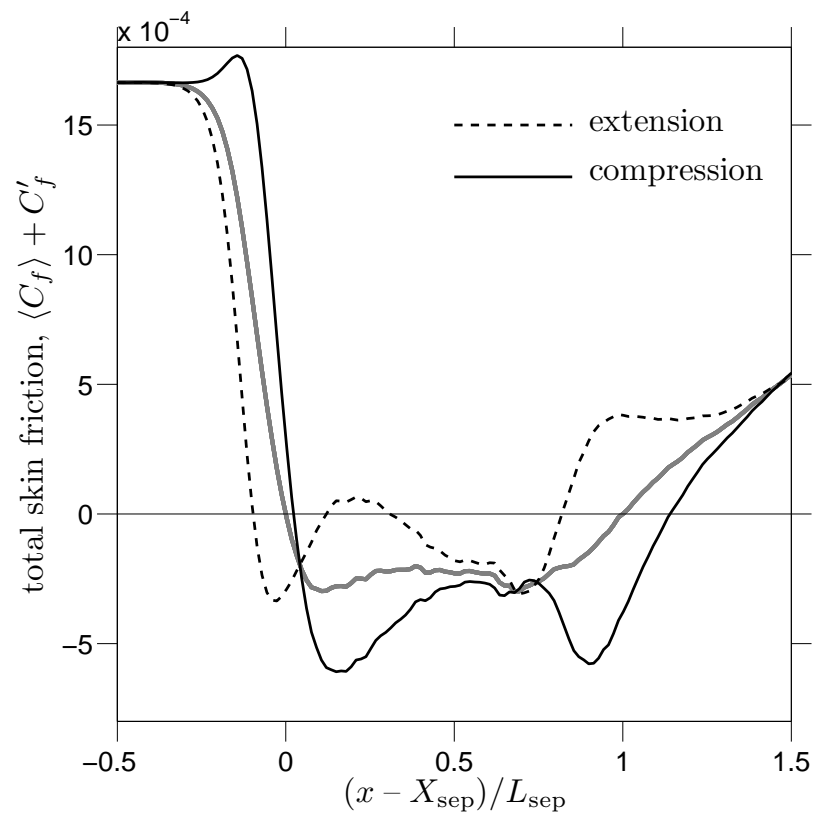

(b)

Figure 15: Global-mode effect on the separation bubble. (a) Global mode fingerprint on the skin friction (curves plotted at different times). (b) Global change in the skin friction (amplified to a non-linear level for demonstration purpose)

growth rates found in this analysis are at most $O\left(0.5 L_{\mathrm{sep}} / U_{\infty}\right)$, which converts to $\omega_{i} \sim O\left(0.1 \delta_{0} / U_{\infty}\right)$ (assuming $L_{\mathrm{sep}} \sim O\left(5 \delta_{0}\right)$ ). This implies that if we consider the case where the initial disturbance was 4 orders of magnitude smaller than the base flow, the linear regime will span a time $\Delta t \approx \ln \left(10^{3}\right) / \omega_{i} \sim O\left(70 U_{\infty} / \delta_{0}\right)$. Therefore, the linear regime involves timescales about two orders of magnitude larger than the characteristic timescale associated with the incoming turbulence. This is consistent with the earlier argument that the stability analysis would be meaningful if it involved timescales larger than the turbulence, so that the turbulence only acts to produce the base flow but does not play a significant role in the development of large-scale motions, just like in the investigations of Gaster et al. [20]. Of course, this does not constitute a proof, but the stability results are found to be consistent with the underlying assumptions made earlier. Based on the results of Gaster et al. [20] and Crouch et al. [7], one cannot rule out the possibility that the aforementioned global mode is meaningful and responsible for the observed low-frequency shock motions in the IUSTI 8-degree shock-reflection case.

\section{Unsteady aspects from the analysis of the LES data}

In this section, we look at unsteady aspects of the LES data. As mentioned earlier, the small-span LES is used for this study as it is the least expensive case to run. The data were obtained as explained in section 2.3. Note that despite the larger bubble length found in the small-span LES, we can still study the unsteady aspects. In fact, Dussauge et al. [13] have shown that the unsteady aspects of the interaction scaled relatively well with the interaction length, so that renormalizing the lengths by the separation length ought to remove the issue related to the use of the small-span LES and its over-predicted separation bubble.

\subsection{Comparison with experimental wall-pressure measurements}

Figure 16 compares the experimental signal (Dupont et al. [11]) with the equivalent LES pressure signal. Both signals were normalized with the upstream pressure and filtered with a $6^{\text {th }}$-order low-pass Butterworth filter with a cutoff Strouhal number of 2, where the Strouhal number is defined as $f L_{\mathrm{sep}} / U_{\infty}$ with $f$ the dimensional frequency. Note that we use the separation-bubble length and not the interaction length. Then, the filtered LES pressure signal was projected by linear interpolation onto the experimental time axis. This allows a direct comparison of the two signals. One can thus see that they share very similar properties, suggesting that the LES must be reproducing the dynamics reasonably well. A closer look at the signals reveals that the experimental signal is slightly richer at frequencies near cutoff, but overall, the resemblance is striking.

Figure 17 compares the two signals in a more rigorous way, from a spectral point of view. Figure 17(a) gives the power spectral density (PSD) of the aforementioned two signals, with an additional LES wall-pressure signal taken upstream of the interaction. This time, the LES signals were not low-pass filtered so that the high-frequency content is retained. However, all signals were segmented using Welch's method (with 50\% overlaps and Hanning windows). Finally, in order to obtain a smooth PSD at high frequencies, we processed the LES signals several times with an increasing number of segments and then reconstructed the entire frequency range to obtain the plots in figure 17. Figure 17(a) confirms the good agreement suggested in figure 16 between the experiment and the LES at 

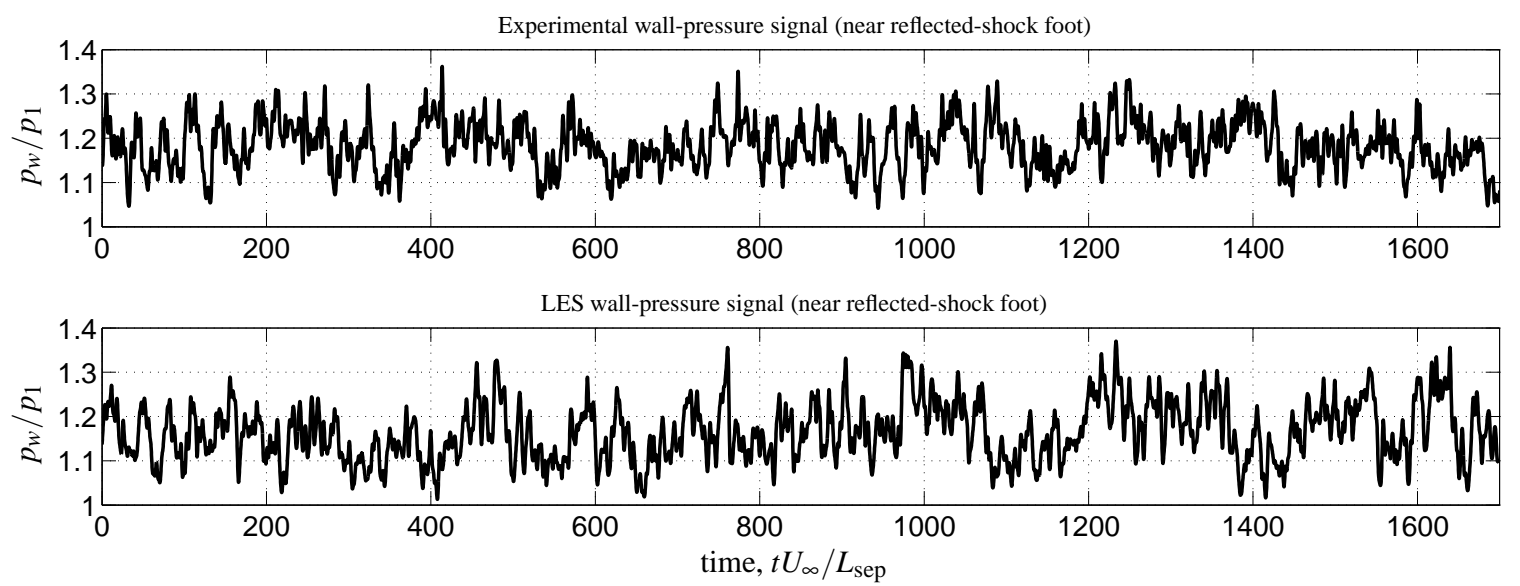

Figure 16: Wall-pressure time signals: experimental and numerical. (Both the LES and experimental data have been filtered with a $6^{\text {th }}$-order low-pass Butterworth filter with a cutoff Strouhal number of 2, and the LES signal has then been projected - via linear interpolation - on the experimental time axis)

low-frequencies. Furthermore, the upstream probe from the LES confirms that the energetic low-frequencies observed near the reflected shock were not introduced by the inlet conditions and thus that the digital-filter approach met our expectations on that aspect.

Figure 17(b) gives the weighted PSD. It is obtained by multiplying the PSD by the frequency (the Strouhal number in our case) and normalizing by the integrated PSD over a given frequency (Strouhal number) range. This representation is convenient to highlight the frequencies which contribute most to the variance of the signal. However, the normalization is arbitrary and one should be careful when comparing the LES and the experiment as the available frequency ranges of the two signals differ. In figure 17(b), we provide an hybrid normalization, labelled "hybrid norm.", where one accounts only for the common frequency range covered between the experimental and LES signals (i.e. between the lowest frequency covered by the LES up to the cutoff frequency of the experimental signal). As shown in figure 17(b), the agreement between the LES and the experiment using the hybrid-frequency range is satisfactory.

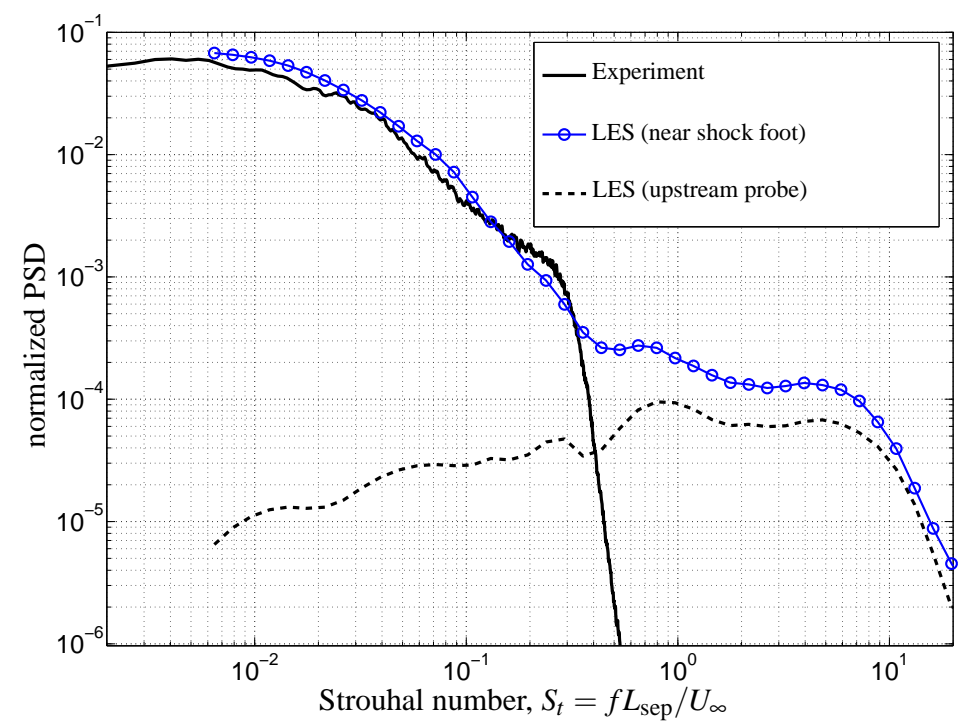

(a) power-spectral density

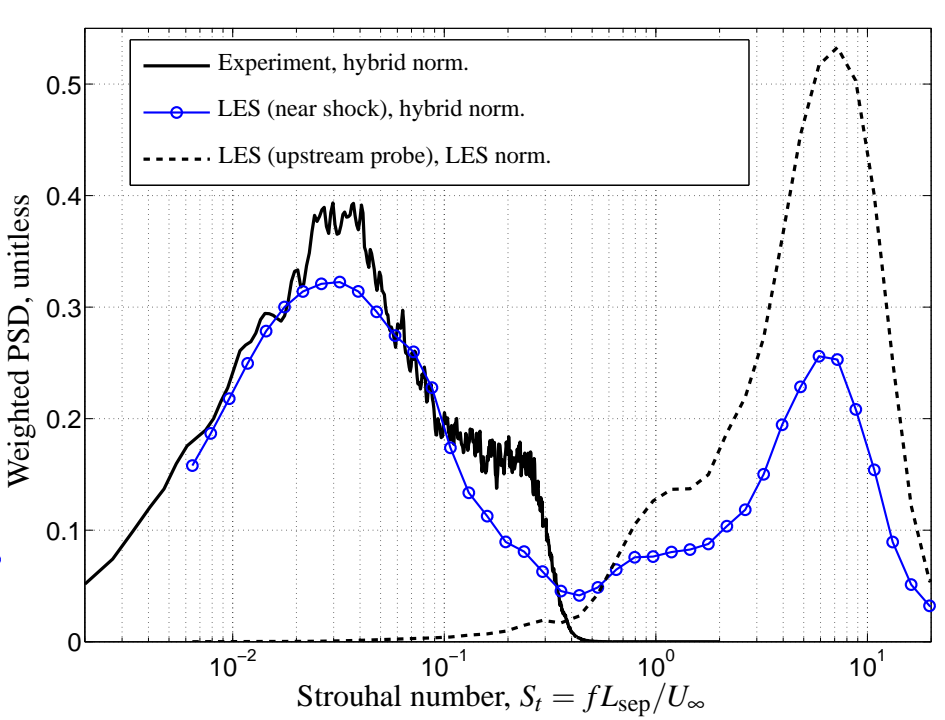

(b) weighted-power-spectral density

Figure 17: Spectral analysis of the wall-pressure signals: experiment vs. LES

These results imply the following. First, that the region under the reflected shock exhibits significant low-frequency oscillations. These so-called low frequencies are broadband and cover at least one frequency decade around $S_{t} \approx 0.03$, giving the reflected shock a very random-like motion. Furthermore, these frequencies are two orders of magnitude smaller than the energetic frequencies related to the turbulence. Since the fluctuations related to the turbulence contribute to the signal-variance nearly as much as the low-frequencies, it makes the distinction between low-frequency and turbulence-related events extremely difficult when looking at a raw time-signal as in figure 16. Second, the good agreement of the LES with the experimental data suggests that the present LES does capture the important dynamics of this interaction: namely, the frequency of the most energetic low-frequency unsteadiness and the bandwidth of the low-frequency content. However, the LES slightly underestimates the amount of energy around $S_{t} \approx 0.3$. Nevertheless, the overall good agreement is an indirect proof that the experimental observations of the existence of a low-frequency content are not due to an artifact of the experimental arrangement.

However, it should be recalled that the present low-frequency analysis is obtained from the narrow-span LES, which was shown to produce a longer separation bubble than expected. One can then wonder why the agreement with the experiment is so good. First, it 
must be recalled that Dussauge et al. [13] have shown that the low-frequency unsteadiness scaled relatively well with the interaction length and it is possible that the narrow-span LES benefits from this choice of lengthscale in the definition of the Strouhal number. In fact, the agreement would be poor if we had used the boundary-layer thickness as the reference lengthscale. Second, assuming that the global mode found in the previous section is related to the low-frequency oscillations, and in light of the experienced sensitivity of the growth rates to the amount of reversed flow, it would not be surprising that the low-frequency oscillations are related to the separation-bubble properties, and more precisely, to the amount of reversed flow, which is related to the bubble height. It was shown earlier that the large-span LES underestimated the bubble height and consequently the magnitude of the reversed flow. Since the narrow-span bubble is longer and taller than the one found in the large-span case, it is likely that the amount of reversed flow in the narrow-span LES closely matches the experiment, artificially leading to a good agreement for the low-frequency dynamics.

\subsection{Upstream influence and digital filter}

Before investigating the wall-pressure fluctuations inside the interaction, we will briefly discuss the upstream influence and the use of the digital-filter approach. Figure 18 shows snapshots of the streamwise velocity-fluctuation field in a plane parallel to the wall at two different altitudes: at $y^{+} \approx 12$ and $y / \delta_{0} \approx 0.2$. The colormap highlights the region of the flow with a velocity deficit. At $y^{+} \approx 12$, we see a very streaky structure. However, the timescales associated with these near-wall turbulence structures are small compared to the timescales associated with the low-frequency shock oscillations. At $y / \delta_{0}=0.2$ (figure 18 (b)) no obvious long-coherent structure is seen. To be more convinced of the absence of such structure in the present LES investigations, one can develop the time history of the velocity fluctuations seen along a numerical wire just before the interaction (corresponding to $x=260 \mathrm{~mm}$ ) and at $y / \delta_{0}=0.2$, as shown in figure 19, where time is converted into space assuming the fluctuations are convected at the local mean velocity $U_{c}$, as in figure 4 in Ganapathisubramani et al. [17] (Taylor's hypothesis). The colormap was designed to highlight any large-scale velocity deficit in the reconstructed flow field. The longest structures one can see are of order $10 \delta_{0}$ long.

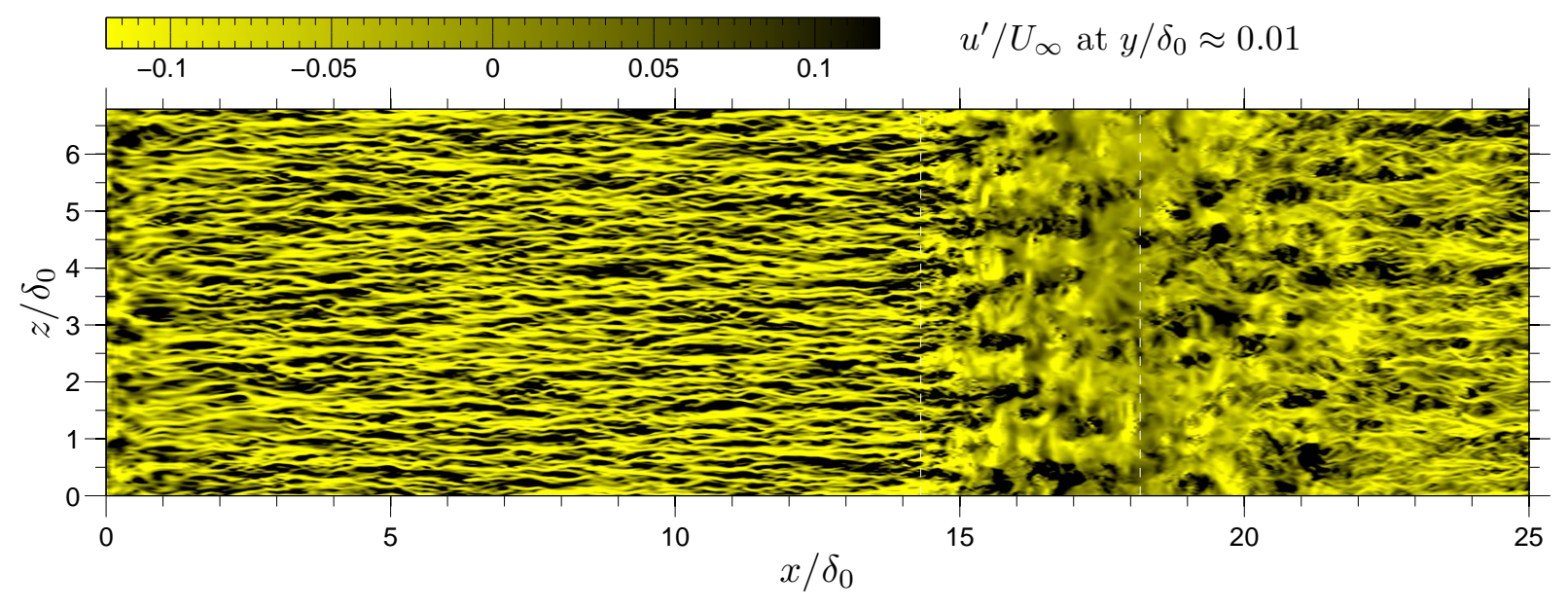

(a) at $y^{+} \approx 12$

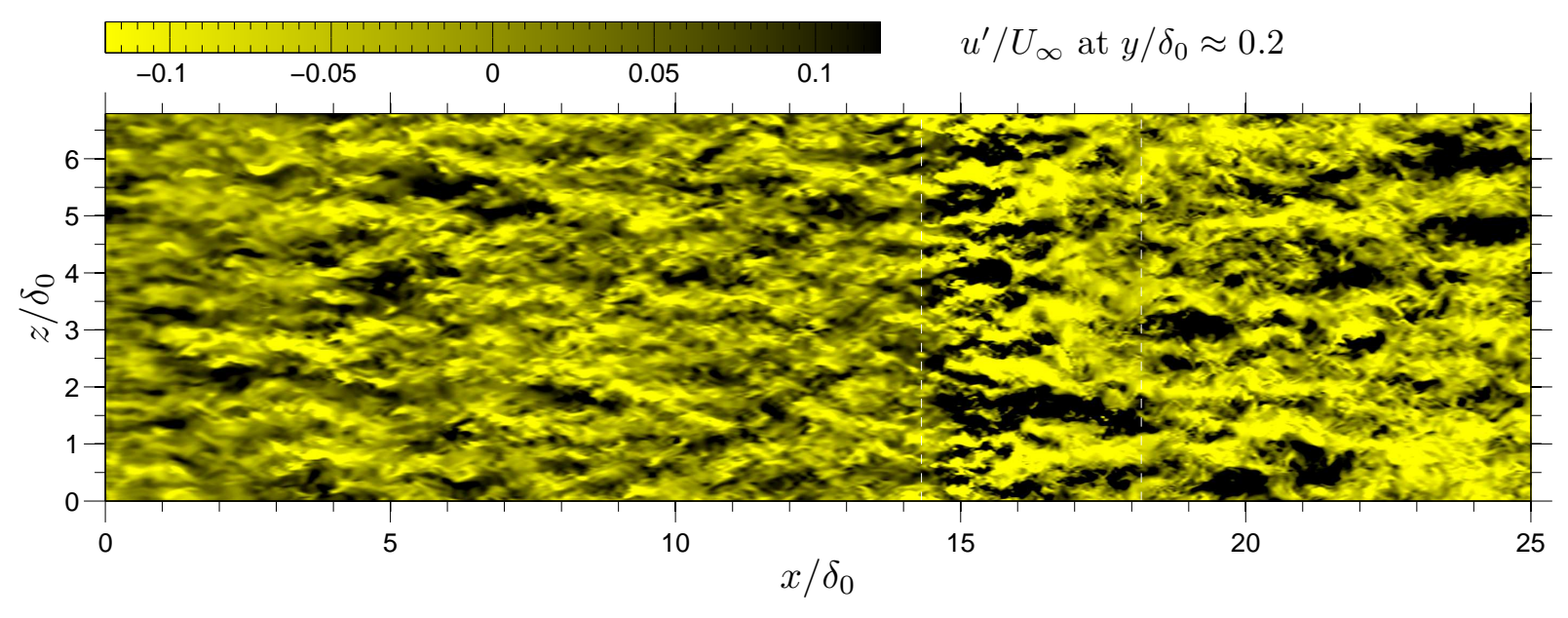

(b) at $y / \delta_{0} \approx 0.2$

Figure 18: Instantaneous snapshot of $u^{\prime} / U_{\infty}$ from the large-span LES case at two different heights

An autocorrelation function (computed from the narrow-span LES at $x=260 \mathrm{~mm}, y / \delta_{0}=0.2$ and in the middle plane of the computational box) is shown in figure 20, where the same time to space transform as in the previous paragraph was applied. Note that the space axis is given on a logarithmic scale to cover long distances. The correlation function is seen to drop to zero in less than 
one boundary-layer thickness. Note that it drops faster than the prescribed correlation in the digital filter. This is expected since we deliberately overestimate the correlation lengthscales to ensure the simulated flow does not relaminarize. Also, it is expected that the correlation function in a turbulent boundary layer drops slightly faster than the prescribed exponential function at the inlet. What is most important to the present study is that the correlation function does remain at zero for large timescales. This was the main objective that motivated the choice of the digital-filter approach, where no cyclic patterns is enforced, as shown by the correlation function. In contrast, the correlation function in Wu and Martin's DNS (figure 4 in [54]) does not drop to zero and does not extend to the period of the recycling/rescaling technique used by the authors.

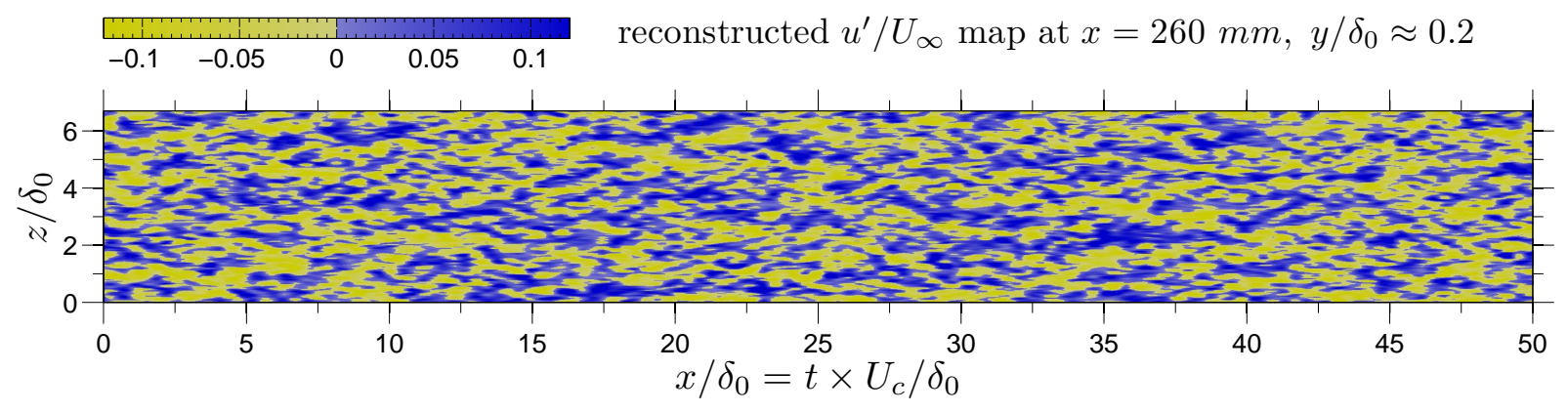

Figure 19: Reconstructed $u^{\prime} / U_{\infty}$ field from a numerical transverse wire located at $x=260 \mathrm{~mm}$ and $y / \delta_{0}=0.2$. $U_{c}$ is the mean streamwise velocity at $x=260 \mathrm{~mm}$ and $y / \delta_{0}=0.2$

From the point of view of the digital filter, we made sure that no structure longer than $O\left(\delta_{0}\right)$ was introduced and this is confirmed by the observed correlation function. However, once inside the computational domain, nothing can prevent larger structures developing, and from figure 19 one can see that structures up to $10 \delta_{0}$ long may develop, corresponding to the size of the available computational domain before interaction. As shown earlier, the narrow-span bubble is about $6 \delta_{0}$ long, while the most energetic low-frequency oscillations are at $S t=f L_{\mathrm{sep}} / U_{\infty} \approx 0.03$. Using the boundary-layer thickness as the lengthscale, the energetic lowfrequency oscillation converts to $f \delta_{0} / U_{\infty} \approx 0.005$. The timescale associated with this frequency is $200 U_{\infty} / \delta_{0}$. Since $U_{c} / U_{\infty} \approx 0.73$, the lengthscale covered during this time using Taylor's hypothesis is $\lambda / \delta_{0} \approx 150$. Using the scaling argument of Ganapathisubramani et al. [18], one would thus need to have $75 \delta_{0}$-long superstructures in the narrow-span LES to explain the observed energetic low-frequency oscillations. This is nearly ten times the size of the longest structures we may have, which makes the "superstructures" or the incoming flow unlikely to be directly responsible for the low-frequency shock motions observed in the present study. This is not to say that upstream disturbances are not important when present in practical applications. We now return to the analysis of the wall-pressure fluctuations inside the interaction.

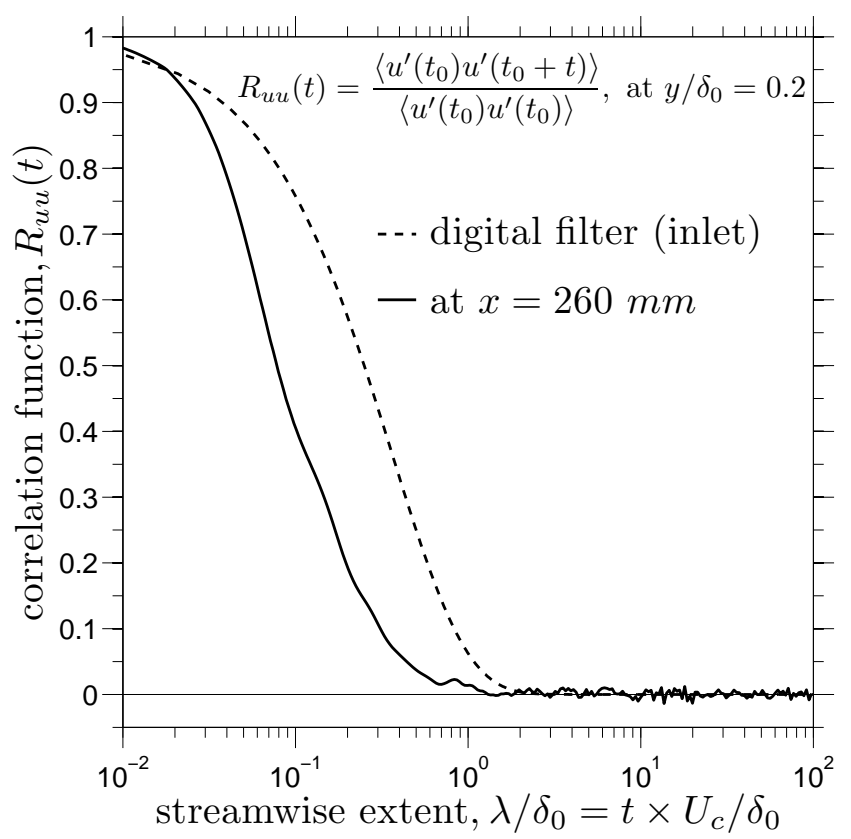

Figure 20: Streamwise-velocity correlation at $x=260 \mathrm{~mm}$ and $y / \delta_{0}=0.2$, compared with the specified correlation at inlet (see equation (28)). $U_{c}$ is the mean streamwise velocity at $x=260 \mathrm{~mm}$ and $y / \delta_{0}=0.2$ 


\subsection{Analysis of a numerical wall-pressure-probe array}

In this section, we further analyze the LES wall-pressure signals from two sets of wall-pressure probes. The first set, which we refer to as the high-spatial/low-time resolution one, is made of the 451 available grid points in the streamwise direction, along the median-line of the computational-box floor, where the pressure was recorded every one hundred iterations. The second set, which we refer as the low-spatial/high-time resolution one, is made of one pressure measurement every five grid points along the same line, but at a sampling rate of one record every ten iterations.

Figure 21(a) is obtained from the low-spatial/high-time resolution array and is simply an extension of figure 17(b) to all the available streamwise locations. The contours are isovalues of the weighted PSD. This is similar to figure 5 in Dupont et al. [11], except that the high-frequency end of the current figure is higher than in Dupont et al.'s [11] paper, owing to the inclusion of the energetically significant high-frequency oscillations related to the turbulence. Figure 21(a) can be interpreted as the map of the most dominant wall-pressure fluctuations as one moves along the streamwise direction. From this point of view, the separation region clearly stands out. More precisely, it is worth noticing that the energetic broadband low-frequency peak mentioned earlier is very localized about the separation point $\left(x=X_{\mathrm{sep}}\right)$. In the remaining part of the separation bubble, the energy is well distributed over three decades of Strouhal numbers. This is in good agreement with Dupont et al. [11]. After the interaction, a new ridge starts forming, similar to the upstream ridge, but at lower Strouhal numbers. This is due to the thicker post-interaction boundary layer, where similar turbulence structures to the upstream boundary-layer ones are produced, but of larger sizes, leaving a similar footprint in the spectrum but at lower Strouhal numbers.

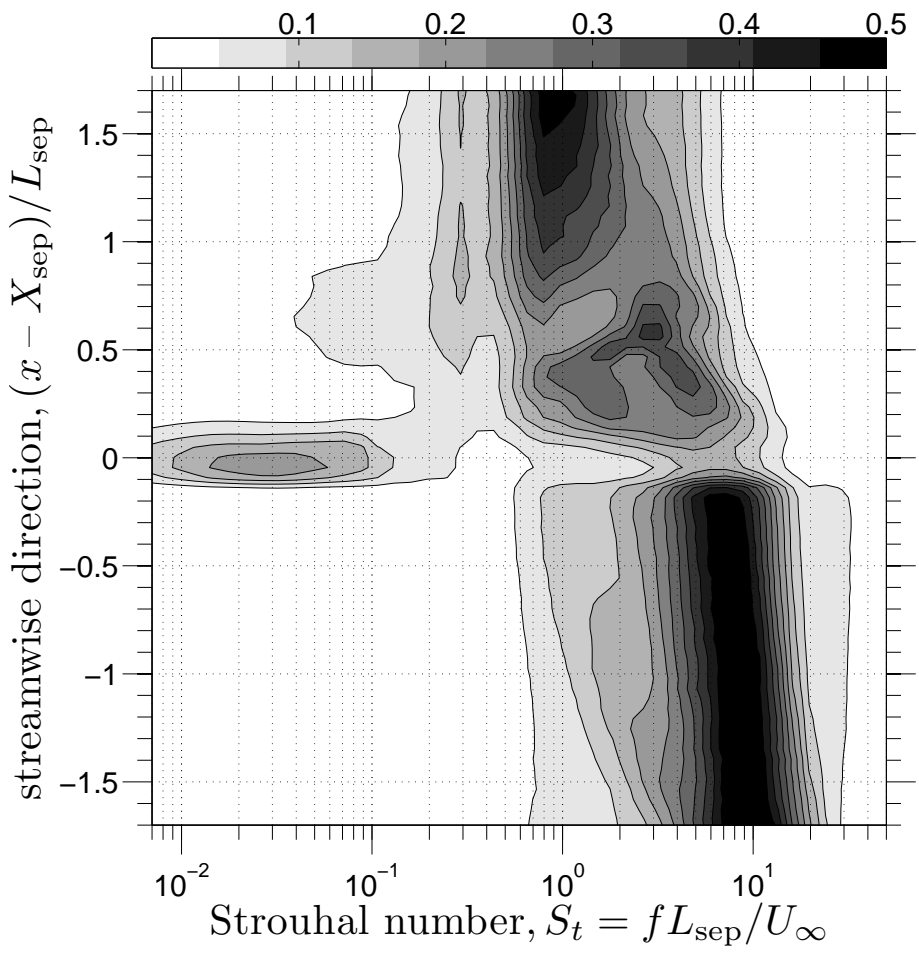

(a) Weighted-power-spectral density map

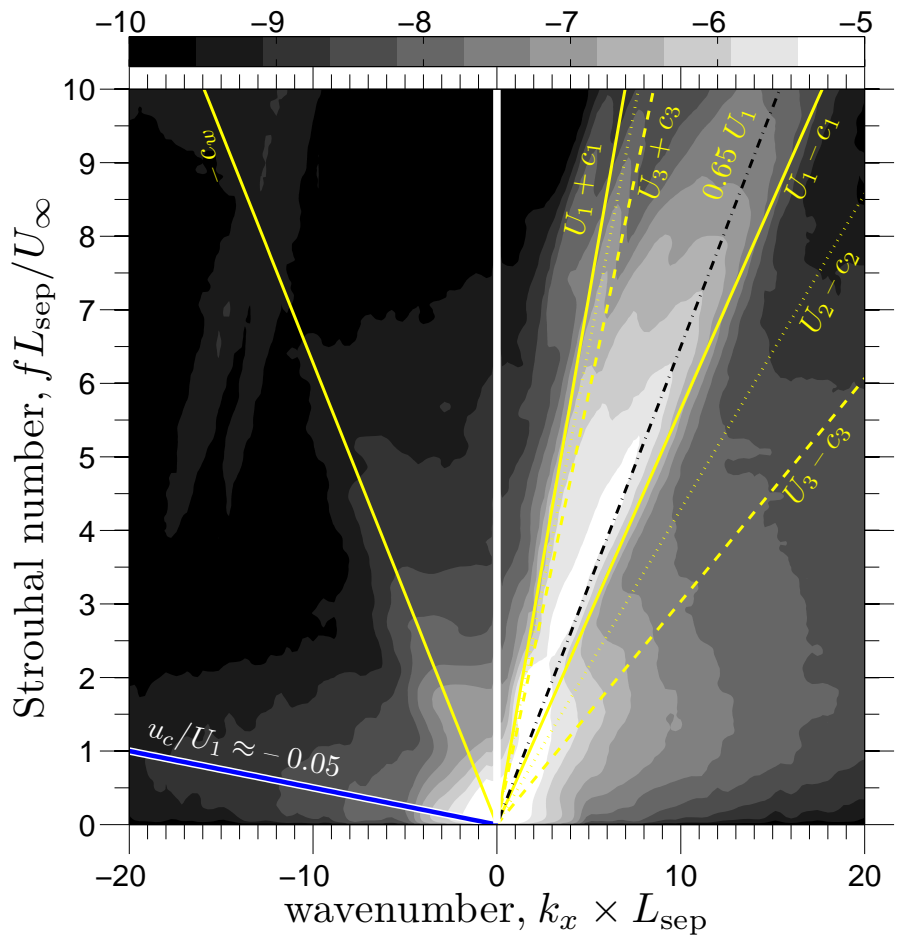

(b) Frequency/wave-number diagram

Figure 21: Energetically significant frequencies as found in the wall-pressure signals. (a) Weighted-power-spectral density map. (b) Frequency/wave-number diagram (where the contour levels - shown on a logarithmic scale - are the premultiplied PSD levels obtained from the two-dimensional Fourier transform of the space-time correlation function of the wall-pressure probe array. The reference probe to compute the space-time correlation function was located at $\left(x-X_{\mathrm{sep}}\right) / L_{\mathrm{sep}}=0.3$ ). The PSDs are premultiplied by (a) the frequency divided by the total resolved power (as in figure 17(b)) for each individual streamwise location, (b) the frequency)

Figure 21(b) was obtained from the high-spatial/low-time resolution array. First, we chose a reference point at $\left(x-X_{\text {sep }}\right) / L_{\text {sep }} \approx 0.3$ and computed the two-point correlation function in space and time. Then, the space-time correlation function was Fourier-transformed in space, with a Hanning window to remove end-effects, followed by a Fourier transform in time to obtain the PSD at different streamwise wavenumbers $k_{x}$, using Welch's method (with 50\% overlaps and Hanning windows). Finally, the PSD was filtered to remove spurious oscillations with a non-causal filter and weighted by the frequency. This gives the frequency/wavenumber diagram shown in figure 21(b) where the contours are the weighted PSD levels. In addition, all possible acoustic dispersion relations are indicated on the figure, where $U_{i}, c_{i}, i \in\{1,2,3\}$ refer to the theoretical potential freestream velocities and local speeds of sound, where region 1 is upstream of interaction, 2 after the incident shock but before the reflected shock and 3 after the interaction. $c_{w}$ is the speed of sound at the wall.

Several observations can be made from figure 21(b). First, on the positive wavenumber side, where a large amount of energy is found for wave speeds ranging from $U_{1}-c_{1}$ to $U_{1}+c_{1}$. Looking more closely at this region, a ridge corresponding to waves propagating at $0.65 U_{1}$ seems to emerge. This ridge is related to the shedding of coherent structures in the shear-layer at the bubble interface. It must 
be recalled that the reference point to build the correlation function was at $\left(x-X_{\mathrm{sep}}\right) / L_{\mathrm{sep}} \approx 0.3$ so that in this case, the shedding of the shear-layer structures at the beginning of the interaction turns out to be the most important contributor of the wall-pressure fluctuations. However, if we had used as the reference point a position upstream of the interaction, the downstream acoustic waves $U_{1}+c_{1}$ would have been relatively more important than the shear-layer structures (this is not shown here). This remark is important to stress that the relative importance (amplitude level) of the structures seen in figure 21(b) depends on the choice of reference point and should thus be considered carefully. However, the structure of the frequency-wavenumber diagram itself does not depend on the location of the reference point within the region considered. In addition to the aforementioned distinct ridges on the positive-wavenumber side, one can see more spatially distributed structures such as the wide lobe for positive wavenumbers which is related to the turbulence-induced pressure fluctuations. The second set of observations concerns the negative-wavenumber side of the figure, where upstream acoustic waves are clearly detected, comforting the possibility of the feedback-loop mechanism proposed by Pirozzoli and Grasso [37]. But of greater interest to the present discussion is the ridge at low frequencies corresponding to upstream-propagating low-frequency waves. A best fit to the ridge gives a convection speed of $-0.05 U_{1}$. Note that replacing the freestream velocity by this convection speed in the definition of the Strouhal number would make the energetic low-frequency oscillations have a Strouhal number of the order of unity.

\subsection{Existence of a phase jump in the wall-pressure fluctuations}

One disadvantage of the frequency/wavenumber diagram (figure 21(b)) is that it cannot tell us where the aforementioned slowlyupstream propagating waves come from. It could, if we were to restrict the streamwise extent on which we perform the analysis and successively move this frame downstream, since past the source point of those waves, we would not find their presence in the diagram anymore. However, this is not a convenient approach. Instead, we prefer to look at the phase evolution of the wall-pressure disturbances at a given frequency. For this, we picked a reference point at $\left(x-X_{\text {sep }}\right) / L_{\text {sep }} \approx-0.2$ and made use of the pressure probes from the high-spatial/low-time resolution array. The results for three different frequencies are given in figure 22 . Note that the phase data were unwrapped so that jumps of $2 \pi$ were removed. In addition, the phase evolution was filtered to remove the noise. One disadvantage of this approach is of course that the obtained phase is contaminated by all streamwise wavenumbers (note that the level of contamination can be estimated from the frequency-wavenumber map described in the previous paragraph).

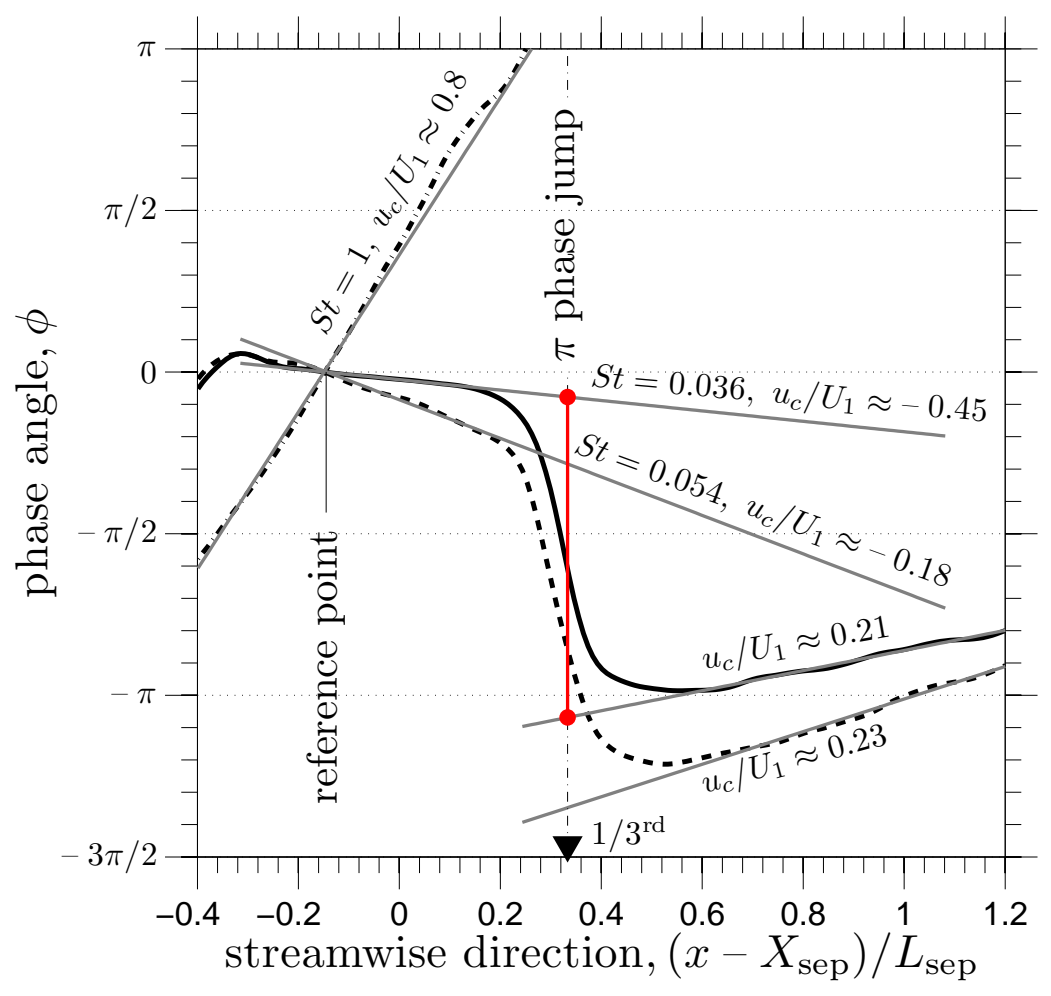

Figure 22: Phase evolution at different frequencies with respect to a reference probe at $\left(x-X_{\text {sep }}\right) / L_{\text {sep }} \approx-0.2$

One can see in figure 22 that for the $S_{t} \approx 1$ case, the phase increases nearly linearly. The convection velocity $U_{c}$ can be deduced from the slope since $U_{c} / U_{\infty}=2 \pi S_{t} /[\mathrm{d} \Phi / \mathrm{d} \xi]$, where $\xi=\left(x-X_{\mathrm{sep}}\right) / L_{\text {sep }}$. However, at lower Strouhal number, we observe interesting changes in the phase evolution, which cannot be explained by the modulo- $2 \pi$ factor. In particular, we find that for $\left(x-X_{\text {sep }}\right) / L_{\text {sep }} \in[-0.3,0.3]$, the phase decreases linearly while it increases linearly everywhere else. Furthermore, the change of slope around $\left(x-X_{\text {sep }}\right) / L_{\text {sep }} \sim 1 / 3$ is abrupt where a phase-jump of about $\pi$ occurs. Before the jump, we find an upstream propagation speed whereas after the jump, we have a downstream propagation speed. This means that the source of the slow-upstream propagating wave discovered in the frequency/wavenumber diagram is located about one third of the way down the bubble. Interestingly, this is 
reminiscent of the global-mode effect on the initial part of the bubble as described in section 4.2.

Hudy et al. [26] have investigated the flow behind a fence by mean of wall-pressure measurements and report the existence of a phase jump of $\pi$ in the middle of the separation bubble, similar to the one observed here. Moreover, the authors suggest that this jump could be related to the presence of a globally unstable mode. Our stability analysis results combined with the above analysis of the LES data seem to argue in favor of such a connection.

\section{Conclusion}

In this paper, we have presented results from several large-eddy simulations of an oblique shock wave generated by an 8 -degree wedge angle impinging on a Mach 2.3 turbulent boundary layer with a Reynolds number of about $2 \times 10^{4}$ (based on the displacement thickness), consistent with the experiments performed at IUSTI $[13,11]$. A modified version of the digital filter approach was implemented to avoid any low-frequency forcing. It was demonstrated that the LES results are in good agreement with the PIV data. Furthermore, the LES grid resolution used $(40.5 \times 1.6 \times 13.5$ in wall units) was shown to produce nearly grid-independent results and the choice of eddy-viscosity model was found to have no noticeable influence on the interaction. The use of a subgrid-scale model, however, was seen to be preferable to an implicit LES approach. By contrast, the choice of domain width was found to strongly influence the interaction length.

Using the time and span-averaged LES data, we performed a linear-stability analysis of the obtained base flow and found globally unstable modes (in the Bi-Global sense) with the most unstable mode being the 2D one, contrary to the 3D global mode found by Robinet [40] in the case of a laminar interaction. In addition, it was shown that the global-mode growth rate are sensitive to the bubble size. Furthermore, this global mode was seen to affect the separation bubble by displacing the separation and reattachment points (in phase) and potentially breaking it up or enhancing the bubble in its upstream section. The timescales involved with such motions were found to be in the order of $10^{2} U_{\infty} / \delta_{0}$, supporting the idea that such a linear-stability analysis is relevant only in the framework of a multi-time scale approach where the globally unstable mode needs to operate at much larger time scales than the underlying boundarylayer turbulence.

A very-long LES run was used to demonstrate the existence of energetic broadband low-frequency motions near the separation point with a peak near $S_{t}=f L_{\mathrm{sep}} / U_{\infty} \approx 0.03$, in excellent agreement with the experimental findings. The success of the digital-filter approach to avoid any particular upstream low-frequency forcing was evidenced and it was explicitly shown that the observed low-frequency shock oscillations were not connected with the inflow turbulence generation, ruling out the possibility of a numerical artifact. This led us to conclude that the low frequencies found in the experiment are also unlikely to be due to an experimental artifact [13].

Upon investigation of the wall-pressure signals, it was further shown that the low frequencies are local to the initial stage of the interaction. Interestingly, some long-wavelength/low-frequency waves propagating upstream were found in the first third of the separation bubble. Upon inspection of the phase evolution at those low frequencies in the LES data, a distinct phase-jump of $\pi$ about one third of the way down the bubble could be observed, reminiscent of the effect the global mode was found to have on the bubble upstream section. Although the relevance of the global mode is not proven, it can be argued that the initial shock displacement could be the result of such a hydrodynamic instability, which would then be competing with the need for momentum balance. The timescales involved in such a mechanism were shown to be about two orders of magnitude larger than the timescales involved in the boundary-layer turbulence, which is consistent with the observed low-frequency motions. Furthermore, those timescales were shown to be strongly modulated by the amount of reversed flow inside the separation bubble. This picture can in principle explain both the low-frequency unsteadiness and its broadband nature.

\section{A The digital filter approach}

Let $\left\{r_{k}\right\}_{1 \leq k \leq p}$ be a set of $p$ random numbers with zero-mean $\left(\overline{r_{k}} \equiv \sum_{k=1}^{p} r_{k} / p=0\right)$ and unit-variance $\left(\overline{r_{k} r_{k}}=\sum_{k=1}^{p} r_{k}^{2} / p=1\right)$. Let $N$ be a positive integer. We define the discrete filter operator $F_{N}$ :

$$
v_{k} \equiv F_{N}\left(r_{k}\right)=\sum_{j=-N}^{N} b_{j} r_{k+j}
$$

where $\left\{b_{j}\right\}_{-N \leq j \leq N}$ is a set of real numbers to be defined later. Noting that the above filter operator is linear, making the averaging and filtering operations commute, and that the set $\left\{r_{k}\right\}_{1 \leq k \leq p}$ is composed of zero-mean and unit-variance random numbers, one can easily show that:

$$
\overline{v_{k}}=0, \text { and } \overline{v_{k} v_{k+q}}=\sum_{j=-N+q}^{N} b_{j} b_{j-q} .
$$

We now model the two-point-correlation function to be of the form (in 1D for simplicity):

$$
R\left(x_{k}+x\right)=\exp \left(-\frac{\pi x}{2 I_{x}}\right),
$$

where $x_{k}$ is a reference point, $x$ some distance away from the reference point and $I_{x}$ a given integral length scale. Note that in the original paper of Klein et al. [30], the authors assume a Gaussian auto-correlation function. By contrast, Xie and Castro [56] argue that auto-correlation functions have a form closer to exponential than Gaussian, hence the current choice in equation (24). This will inevitably produce an energy-decay rate of -2 in place of the expected $-5 / 3$ law. However, one can argue that this choice of function is 
correct for the large-scale structures and that most of the discrepancies will occur at the smallest scales [56], which are found to recover the modeling errors more rapidly than the large-scale structure.

On the computational grid, if we define $n$ such that $I_{x}=n \Delta x$ and $x=q \Delta x$, equation (24) becomes:

$$
R\left(x_{k}+q \Delta x\right) \equiv \frac{\overline{v_{k} v_{k+q}}}{\overline{v_{k} v_{k}}}=\exp \left(-\frac{\pi q}{2 n}\right) .
$$

Using equation (23), one finds that the filter coefficients can be computed by solving the following system:

$$
\frac{\sum_{j=-N+q}^{N} b_{j} b_{j-q}}{\sum_{j=-N}^{N} b_{j}^{2}}=\exp \left(-\frac{\pi q}{2 n}\right) .
$$

The solution is approximated by:

$$
b_{k} \approx \frac{\tilde{b}_{k}}{\left(\sum_{j=-N}^{N} \tilde{b}_{j}^{2}\right)^{1 / 2}} \quad \text { with: } \tilde{b}_{k}=\exp \left(-\frac{\pi k}{n}\right) .
$$

Klein et al. [30] find this to be a good approximation if $N \geq 2 n$ (which is not thought to be a computationally expensive requirement).

Upon application of the $F_{N}$-operator with the above definition for the convolution coefficients, the initial random field of zero-mean and unit-variance has been given a coherence integral length scale $I_{x}$. Note that the above 1D description can be extended to 2D by simply defining the $2 \mathrm{D}$ convolution coefficients as: $b_{j k}=b_{j} b_{k}$. This completes the main tools for the digital-filter approach. Let us now describe the step-by-step procedure in order to produce the inlet flow-variable fluctuations.

First, we choose the integral length scales $I_{x}, I_{y}, I_{z}$ that we want to prescribe. Given the grid spacing, we convert these into an equivalent number of grid points, i.e. $n_{I_{x}}=I_{x} / \Delta x$. This sets the filter size: $N_{F_{x}}=2 n_{I_{x}}$. In the current implementation, we make use of a zonal approach by defining a different set of filters in the near-wall region and the outer-region, as in Veloudis et al. [52]. Then, we prepare the convolution coefficients $\left\{b_{k}\right\}-N_{F_{x}} \leq k \leq N_{F_{x}}$ using equation (27) (in 1D).

Next, we generate a set of random numbers with a normal distribution about zero and unit variance. Since we need to generate a large number of these sets of random numbers, it is worth noting the following improvement over the commonly used approach to obtain a normal distribution. Most pseudo-random number generators will generate uniformly distributed numbers and one usually achieves a normal distribution simply by adding many (12 in Xie and Castro [56]) of those uniformly distributed sets. However, using the Box-Muller theorem, one only needs two sets: if $a$ and $b$ are two independent numbers uniformly distributed in $(0,1]$, combining them such that $c=\sqrt{-2 \ln (a) \cos (2 \pi b)}$ and $d=\sqrt{-2 \ln (a) \sin (2 \pi b)}$ will make $c$ and $d$ be two independent numbers from a normal distribution of unit-standard deviation.

Once we have the normally distributed random numbers with zero-mean and unit-variance, we filter them using the convolution coefficients computed previously: $v_{k} \equiv F_{N_{F_{x}}}\left(r_{k}\right)=\sum_{j=-N_{F_{x}}}^{N_{F_{x}}} b_{j} r_{k+j}$ (in 1D). The "velocity" field $v_{k}$ now has the prescribed length scale $I_{x}$. Next we correlate the newly computed field $v_{k}$ with the previous one, $v_{k}^{\text {old }}$. (Except of course when performing the very first time step.) The following formula was suggested by Xie and Castro [56]. It avoids the filtering of a 3D field as originally proposed by Klein et al. [30].

$$
\rho_{k}=v_{k}^{\text {old }} \exp \left(-\frac{\pi \Delta t}{2 \tau}\right)+v_{k} \sqrt{1-\exp \left(-\frac{\pi \Delta t}{\tau}\right)},
$$

where $\Delta t$ is the time step and $\tau$ is the Lagrangian time scale $\left(\tau=I_{x} / U\right.$ in the present calculations, where $U$ and $I_{x}$ are the prescribed inlet mean streamwise velocity profile and integral length scale, respectively). The field $\rho_{k}$ now contains all the enforced two-point correlation functions as well as the prescribed streamwise correlations thanks to equation (28). The single-point correlations can now be specified, as originally proposed by Lund et al. [33]. In 3D, it can be written:

$$
\left[\begin{array}{c}
u(0, y, z, t) \\
v(0, y, z, t) \\
w(0, y, z, t)
\end{array}\right]=\underbrace{\left[\begin{array}{c}
\langle u(0, y, z)\rangle \\
\langle v(0, y, z)\rangle \\
\langle w(0, y, z)\rangle
\end{array}\right]}_{U_{i}(0, y, z)}+\underbrace{\left[\begin{array}{ccc}
\sqrt{R_{11}} & 0 & 0 \\
R_{21} / \sqrt{R_{11}} & \sqrt{R_{22}-\left(R_{21} / \sqrt{R_{11}}\right)^{2}} & 0 \\
0 & 0 & \sqrt{R_{33}}
\end{array}\right]\left[\begin{array}{c}
\rho^{u}(y, z) \\
\rho^{v}(y, z) \\
\rho^{w}(y, z)
\end{array}\right]}_{u_{i}^{\prime}(0, y, z, t)},
$$

where $\left\{R_{i j}\right\}_{(i, j) \in\{1,2,3\}}$ is the prescribed Reynolds-stress tensor.

We thus have built the inflow time-dependent velocity field. We are left with the thermodynamic variables. To generate the thermodynamic fluctuations, we make use of the previously determined velocity perturbations $u_{i}^{\prime}$, invoking the Strong Reynolds Analogy (SRA):

$$
\frac{T^{\prime}}{\bar{T}}=-(\gamma-1) \mathrm{Ma}^{2} \frac{u^{\prime}}{U}, \text { with } \mathrm{Ma}^{2}=M^{2} \frac{U^{2}}{\bar{T}},
$$

where $\bar{T}$ is the local mean temperature. The validity of the SRA is debatable. In fact, from Guarini et al.'s DNS [23], we know that the above equation is wrong in general. However, equation (30) is also shown in Guarini et al.'s DNS [23] to be correct in a weaker sense, that it provides the correct RMS correlation. Recently, Martin [34] obtained good results using the SRA as a mean to initialize the flow in a DNS. The use of the SRA is thus believed to be acceptable as a first approach.

Once $T^{\prime}$ is computed from the above equation, assuming that the pressure is constant across the boundary layer (invoking the boundary-layer approximation) and that the pressure fluctuations are negligible compared to the velocity, density and temperature fluctuations (an hypothesis already used in the SRA), one finds that:

$$
\frac{\rho^{\prime}}{\bar{\rho}}=-\frac{T^{\prime}}{\bar{T}}
$$


All the variables are thus prescribed at the inflow and one goes through the above procedure again at the following time step. In our simulations, we make use of the Mersenne Twister [35] generator to obtain the uniformly distributed random numbers. Using the Box-Muller theorem, each time step will thus require two sets of $3 \times N_{y} \times N_{z}$ random numbers (3 components in the inflow plane with $N_{y}, N_{z}$ in the order of $100-N_{y}$ and $N_{z}$ are the number of grid points in the wall-normal and spanwise directions, respectively). Given the Mersenne Twister generator period of $2^{19937}-1$, we are guaranteed not to introduce any cyclic behavior in the computational domain. In addition, the combined use of the Box-Muller theorem, the Mersenne Twister generator and the 2D filtering approach of Xie and Castro [56] produce an efficient method which was not found to be slower than the analytical approach of Sandham et al. [43].

Acknowledgements The authors would like to acknowledge the UK Turbulence Consortium EP/D044073/1 for the computational time provided on the HPCx and HECToR facilities, the UK's national high-performance computing service, which is provided by EPCC at the University of Edinburgh and by CCLRC Daresbury Laboratory, and funded by the Office of Science and Technology through EPSRC's High End Computing Program. We are also grateful to the University of Southampton for the access to its high-performance computer, Iridis2. In addition, we would like to acknowledge the financial support of the European Union through the Sixth Framework Program with the UFAST project (www . ufast.gda.pl). Finally, we are grateful to J.-P. Dussauge and his co-workers at IUSTI, as well as Eric Garnier at ONERA, for kindly making their data available.

\section{References}

[1] Adams, N. A. DNS of Shock Boundary-Layer Interaction - Preliminary Results for Compression Ramp Flow. CTR Annual Research Briefs 1997, Center for Turbulence Research, Stanford University and NASA Ames Research Center, Stanford, California.

[2] Adams, N. A. Direct Simulation of the Turbulent Boundary Layer along a Compression Ramp at $M=3$ and $\operatorname{Re}_{\theta}=1685$. J. Fluid Mech., 420:47-83, 2000.

[3] Adamson Jr., T. C., Messiter, A. F. Analysis of Two-Dimensional Interactions between Shock Waves and Boundary Layers. Ann. Rev. Fluid Mech., 12:103-38, 1980.

[4] Andreopoulos, J., Muck, K. D. Some New Aspects of the Shock Wave/ Boundary Layer Interaction in Compression Ramp Flows. J. Fluid Mech., 180:405-428, 1987.

[5] Beresh, S. J., Clemens, N. T., Dolling, D. S. Relationship Between Upstream Turbulent Boundary-Layer Velocity Fluctuations and Separation Shock Unsteadiness. AIAA Journal, 40(12), 2002.

[6] Carpenter, M. H., Nordstrom, J., Gottlieb, D. A Stable and Conservative Interface Treatment of Arbitrary Spatial Accuracy. NASA/CR-1998-206921, February 1998, 1998.

[7] Crouch, J. D., Garbaruk, A., Magidov, D. Predicting the onset of flow unsteadiness based on global instability. Journal of Computational Physics, 224:924-940, 2007.

[8] Dennis, D. J. C., Nickels, T. B. On the limitations of Taylor's hypothesis in constructing long structures in a turbulent boundary layer. J. Fluid Mech., 614:197-206, 2008.

[9] Dolling, D. S. Fifty Years of Shock-Wave/Boundary-Layer Interaction Research: What Next? AIAA Journal, 39(8), 2001.

[10] Ducros, F., Ferrand, V., Nicoud, F., Weber, C., Darracq, D., Gacherieu, C., Poinsot, T. Large-Eddy Simulation of the Shock/Turbulence Interaction. Journal of Computational Physics, 152:517-549, 1999.

[11] Dupont, P., Haddad, C., Debiève, J. F. Space and Time Organization in a Shock-Induced Separated Boundary Layer. J. Fluid Mech., 559:255-277, 2006.

[12] Dupont, P., Piponniau, S., Sidorenko, A., Debiève, J. F. Investigation by Particle Image Velocimetry Measurements of Oblique Shock Reflection with Separation. AIAA Journal, 46(6), 2008.

[13] Dussauge J.-P., Dupont P., Debiève J.-F. Unsteadiness in Shock Wave Boundary Layer Interaction with Separation. Aerospace Science and Technology, 10:85-91, 2006.

[14] Dussauge, J.-P., Piponniau, S. Shock/boundary-layer interactions: Possible sources of unsteadiness. Journal of Fluids and Structures, 24:1166-1175, 2008.

[15] Eckert, E. R. G. Engineering Relations for Friction and Heat Transfer to Surfaces in High Velocity Flow. Journal of the Aeronautical Sciences, pages 585-587, 1955.

[16] Erengil, M. E., Dolling, D. S. Physical Causes of Separation Shock Unsteadiness in Shock-Wave/Turbulent Boundary-Layer Interactions. AIAA Paper 93-3134, 1993.

[17] Ganapathisubramani, B., Clemens, N. T., Dolling, D. S. Effect of upstream coherent structures on low-frequency motion of shock-induced turbulent separation. In 45 ${ }^{\text {st }}$ Aerospace Sciences Meenting and Exhibit, number AIAA paper $2007-1141,2007$. 
[18] Ganapathisubramani, B., Clemens, N. T., Dolling, D. S. Effects of Upstream Boundary Layer on the Unsteadiness of ShockInduced Separation. J. Fluid Mech., 585:369-394, 2007.

[19] Garnier, E., Sagaut, P., Deville, M. Large Eddy Simulation of Shock/Boundary-Layer Interaction. AIAA Journal, 40(10), 2002.

[20] Gaster, M., Kit, E., Wygnanski, I. Large-scale structures in a forced turbulent mixing layer. J. Fluid Mech., 150:23-39, 1985.

[21] Germano, M., Piomelli, U., Moin, P., Cabot, W. A Dynamic Subgrid-Scale Eddy Viscosity Model. Physics of Fluids, 3(7):1760$1765,1991$.

[22] Germano, M., Piomelli, U., Moin, P., Cabot, W. Erratum: A Dynamic Subgrid-Scale Eddy Viscosity Model. Physics of Fluids, 3(12):3128-3128, 1991.

[23] Guarini, S. E., Moser, R. D., Shariff, K., Wray, A. Direct Numerical Simulation of a Supersonic Turbulent Boundary Layer at Mach 2.5. J. Fluid Mech., 414:1-33, 2000.

[24] Hou, Y. X., Clemens, N. T., Dolling, D. S. Wide-Field PIV Study of Shock- Induced Turbulent Boundary Layer Separation. In $41^{\text {st }}$ Aerospace Sciences Meenting and Exhibit, number AIAA paper 2003-0441, 2003.

[25] Huang, P. G., Coleman, G. N., Bradshaw, P. Compressible Turbulent Channel Flows: DNS Results and Modelling. J. Fluid Mech., 305:185-218, 1995.

[26] Hudy, L. M., Naguib, A. M., Humphreys, W. M. Jr. Wall-Pressure Measurements Beneath a Separating/Reattaching Flow Region. Physics of Fluids, 15(3), 2003.

[27] Inagaki, M, Kondoh, T., Nagano, Y. A Mixed-Time-Scale SGS Model With Fixed Model-Parameters for Practical LES. Journal of Fluids Engineering, 127:1-13, January 2005.

[28] Katzer, E. On the Lengthscales of Laminar Shock/Boundary-Layer Interaction. J. Fluid Mech., 206:477-496, 1989.

[29] Keating, A., Piomelli, U., Balaras, E., Kaltenbach, H.-J. A Priori and A Posteriori Tests of Inflow Conditions for Large-Eddy Simulation. Physics of Fluids, 16(12), 2004.

[30] Klein, M., Sadiki, A., Janicka, J. A Digital Filter Based Generation of Inflow Data for Spatially Developing Direct Numerical or Large Eddy Simulations. Journal of Computational Physics, 186:652-665, 2003.

[31] Li, Q. Numerical Study of Mach Number Effects in Compressible Wall-Bounded Turbulence. PhD thesis, School of Engineering Sciences, University of Southampton, June 2003.

[32] Loginov, M. S., Adams, N. A., Zheltovodov, A. A. Large-Eddy Simulation of Shock-Wave/Turbulent-Boundary-Layer Interaction. J. Fluid Mech., 565:135-169, 2006.

[33] Lund, T. S., Wu, X., Squires, K. D. Generation of Turbulent Inflow Data for Spatially-Developing Boundary Layer Simulations. Journal of Computational Physics, 140:233-258, 1998.

[34] Martin, M. P. Direct Numerical Simulation of a Hypersonic Turbulent Boundary Layers. Part 1. Initialization and Comparison with Experiments. J. Fluid Mech., 570:347-364, 2007.

[35] Matsumoto, M., Nishimura, T. Mersenne Twister: A 623-Dimensionally Equidistributed Uniform Pseudorandom Number Generator. ACM Trans. on Modeling and Computer Simulation, 8(1):3-30, 1998.

[36] Moin, P., Squires, K., Cabot, W., Lee, S. A Dynamic Subgrid-Scale Model for Compressible Turbulence and Scalar Transport. Physics of Fluids, 3(11):2746-2757, 1991.

[37] Pirozzoli S., Grasso F. Direct Numerical Simulation of Impinging Shock Wave/Turbulent Boundary Layer Interaction at $M=2.25$. Physics of Fluids, 18(6), 2006.

[38] Plotkin, K. J. Shock Wave Oscillation Driven by Turbulent Boundary-Layer Fluctuations. AIAA Journal 1036-1040, 13, 1975.

[39] Ringuette, M. J., Wu, M., Martin, M. P. Coherent structures in direct numerical simulation of supersonic turbulent boundary layers at Mach 3. J. Fluid Mech., 594:59-69, 2008.

[40] Robinet, J.-Ch. Bifurcations in Shock-Wave/Laminar-Boundary-Layer Interaction: Global Instability Approach. J. Fluid Mech., 579:85-112, 2007.

[41] Sagaut, P., Garnier, E., Tromeur, E., Larchevêque, L., Labourasse, E. Turbulence Inflow Conditions for Large-Eddy Simulation of Compressible Wall-Bounded Flows. AIAA Journal, 42(3), 2004.

[42] Sandham, N. D., Li, Q., Yee, H. C. Entropy Splitting for High-Order Numercial Simulation of Compressible Turbulence. Journal of Computational Physics, 178:307-322, 2002.

[43] Sandham, N. D., Yao, Y. F., Lawal, A. A. Large-Eddy Simulation of Transonic Turbulent Flow over a Bump. International Journal of Heat and Fluid Flow, 24:584-595, 2003. 
[44] Sandhu, H. S., Sandham, N. D. Boundary Conditions for Spatially Growing Compressible Shear Layers. Technical Report QMWEP-1100, Queen Mary University of London, 1994, 1994.

[45] Spalart, P. R. Direct Simulation of a Turbulent Boundary Layer up to $\operatorname{Re}_{\theta}=1410$. J. Fluid Mech., $187,1988$.

[46] Stewartson, K., Williams, P. G. Self-induced Separation. Proceedings of the Royal Society of London. Series A, Mathematical and Physical Sciences, 312:281-206, 1969.

[47] Teramoto S. Large-Eddy Simulation of Transitional Boundary Layer with Impinging Shock Wave. AIAA Journal, 43(11), 2005.

[48] K. W. Thompson. Time Dependent Boundary Conditions for Hyperbolic Systems. Journal of Computational Physics, 68(1):1-24, 1987.

[49] Touber, E., Sandham, N. D. Oblique Shock Impinging on a Turbulent Boundary Layer: Low-Frequency Mechanisms. In $38^{\text {th }}$ Fluid Dynamics Conference and Exhibit, number AIAA paper 2008-4170, 2008.

[50] Ünalmis, O. H., Dolling, D. S. Decay of Wall Pressure Field and Structure of a Mach 5 Adiabatic Turbulent Boundary Layer. AIAA Paper 94-2363, 1994.

[51] van Driest, E. R. On Turbulent Flow Near a Wall. AIAA Journal Special Supplement: Centennial of Powered Flight, 23(11), 1956.

[52] Veloudis, I., Yang, Z., McGuirk, J. J., Page, G. J., Spencer, A. Novel Implementation and Assessment of a Digital Filter Based Approach for the Generation of LES Inlet Conditions. Flow, Turbulence and Combustion, 74(1):1-24, 2007.

[53] Vreman, A. W. Direct and Large-Eddy Simulation of the Compressible Turbulent Mixing Layer. PhD thesis, Department of Applied Mathematics, University of Twente, November 1995.

[54] Wu, M., Martin, M. P. Direct Numerical Simulation of Supersonic Turbulent Boundary Layer over a Compression Ramp. AIAA Journal, 45(4), 2007.

[55] Wu, M., Martin, M. P. Analysis of shock motion in shockwave and turbulent boundary layer interaction using direct numerical simulation data. J. Fluid Mech., 594:71-83, 2008.

[56] Xie, Z. T., Castro, I. P. Efficient Generation of Inflow Conditions for Large-Eddy Simulation of Street-Scale Flows. Flow, Turbulence and Combustion, 81(3):449-470, 2008.

[57] Yee, H. C., Sandham, N. D., Djomehri, M. J. Low-Dissipative High-Order Shock-Capturing Methods Using Characteristic-Based Filters. Journal of Computational Physics, 150:199-238, 1999. 